bioRxiv preprint doi: https://doi.org/10.1101/845230; this version posted November 16,2019 . The copyright holder for this preprint (which was not certified by peer review) is the author/funder, who has granted bioRxiv a license to display the preprint in perpetuity. It is made available under aCC-BY-NC-ND 4.0 International license.

\title{
Nova proteins direct synaptic integration of somatostatin interneurons through activity- dependent alternative splicing
}

Brie Wamsley ${ }^{1,3,7+}$, Leena A. Ibrahim ${ }^{3,4,7}$, Nusrath Yusuf ${ }^{1,3,4}$, Elaine Fisher ${ }^{3,4}$, Xavier Hubert Jaglin ${ }^{1,3}$, Qing Xư ${ }^{5}$, Lihua Guo ${ }^{5}$, Alireza Khodadadi-Jamayran², Emilia Favuzzi ${ }^{3,4}$, Yuan Yuan ${ }^{6}$, Jordane Dimidschstein ${ }^{4}$, Robert Darnell ${ }^{6}$, and Gord Fishell $1,3,4, \#$

\footnotetext{
1 NYU Neuroscience Institute and the Department of Neuroscience and Physiology; Smilow Research Center, New York University School of Medicine, 522 First Avenue; New York, NY 10016, USA.

2 Genome Technology Center, Applied Bioinformatics Laboratories, NYU Langone Medical Center; 550 First Avenue, MSB 304 ; New York, NY 10016, USA.

${ }^{3}$ Department of Neurobiology, Harvard Medical School, 220 Longwood Ave., Boston, MA 02115

${ }^{4}$ Stanley Center at the Broad, 75 Ames St., Cambridge, MA 02142

${ }^{5}$ Center for Genomics \& Systems Biology, New York University, Abu Dhabi, UAE

${ }^{6}$ Laboratory of Molecular Neuro-Oncology, The Rockefeller University, New York, NY

7 These authors contributed equally

+Current address: Department of Neurology, David Geffen School of Medicine, UCLA, Los Angeles, CA, USA

\# Lead and corresponding author: gordon_fishell@hms.harvard.edu
} 


\section{Abstract}

Somatostatin interneurons are the earliest born population of inhibitory cells. They are crucial to support normal brain development and function; however, the mechanisms underlying their integration into nascent cortical circuitry are not well understood. In this study, we begin by demonstrating that the maturation of somatostatin interneurons is activity dependent. We then investigated the relationship between activity, alternative splicing and synapse formation within this population. Specifically, we discovered that the Nova family of RNA-binding proteins are activity-dependent and are essential for the maturation of somatostatin interneurons, as well as their afferent and efferent connectivity. Moreover, in somatostatin interneurons, Nova2 preferentially mediates the alternative splicing of genes required for axonal formation and synaptic function. Hence, our work demonstrates that the Nova family of proteins are centrally involved in coupling developmental neuronal activity to cortical circuit formation. Keywords: Interneuron; Neurodevelopment; Activity; Alternative splicing; Connectivity; Synapse; Nova1; Nova2. 


\section{INTRODUCTION}

Somatostatin cortical interneurons (SST cINs) constitute $\sim 30 \%$ of all inhibitory interneurons in the cerebral cortex. They are crucial for gating the flow of the sensory, motor, and executive information necessary for the proper function of the mature cortex (Fishell and Rudy, 2011; Kepecs and Fishell, 2014; Tremblay et al., 2016). In particular, Martinotti SST cINs, the most prevalent SST cIN subtype, are present in both the infragranular and supragranular layers of the cortex and extend their axons into Layer 1 (L1) (Lim et al., 2018; Muñoz et al., 2017; Nigro et al., 2018). They specifically target the distal dendrites of neighboring excitatory neurons, thus providing the feedback inhibition necessary for modulating dendritic integration (Adler et al., 2019; Favuzzi et al., 2019; Kapfer et al., 2007; Silberberg and Markram, 2007). These roles are dependent upon the remarkable ability of SST cINs to form specific synaptic connections with select excitatory and inhibitory cell types during development.

The mechanisms responsible for generating the precise functional connectivity of SST cINs are poorly understood. Early neuronal activity has emerged as an important cue in directing the maturation of cINs (Wamsley and Fishell, 2017). In addition, recent work has implicated activity as being centrally involved in alternative splicing (Eom et al., 2013; Furlanis and Scheiffele, 2018; lijima et al., 2011a; Lee et al., 2007; 2009; Mauger et al., 2016; Quesnel-Vallières et al., 2016; Vuong et al., 2016; 2018; Xie and Black, 2001). However, whether these processes are coupled within interneurons has not been explored.

The Nova family of RNA-binding proteins (Nova1 and Nova2) have been shown to control the splicing and stability of transcripts encoding a variety of neurotransmitter receptors, ion channels, and transmembrane cell adhesion molecules known to affect synaptogenesis and excitability (Dredge and Darnell, 2003; Eom et al., 2013; Saito et al., 2016; 2019; Ule et al., 2005; 2003a; 2006; Yano et al., 2010). Notably both Nova1 and Nova2 are strongly expressed within clNs during the period of synaptogenesis and as such represent promising effectors that may direct the maturation of SST cINs.

Here we report that neuronal activity is vital for the proper establishment of both afferent and efferent SST cIN connectivity. We show that the conditional loss of Nova1 or Nova2 largely 
phenocopies the effect of dampening activity during circuit assembly, leading to a loss of excitatory synaptic afferents onto SST cINs, as well as their efferent inhibitory output. At a molecular level these changes are mediated by a Nova-dependent program, which controls the alternative splicing of mRNAs encoding for pre- and post-synapse proteins. Demonstrating a direct link between activity, Nova function and synaptogenesis, overexpression of Nova2 within SST cINs dramatically increases synaptogenesis, a phenotype that can be suppressed by damping neuronal activity within these cells. Thus, our work indicates that early activity is required for the proper establishment of SST cIN connectivity and maturation through a Nova-dependent mechanism. 


\section{RESULTS}

\section{Neuronal activity affects the synaptic development of SST cINs}

The cortex exhibits a variety of dynamic network activity patterns during cortical synaptogenesis (Allene and Cossart, 2010; Garaschuk et al., 2000; J. W. Yang et al., 2009). These are comprised by both spontaneous and sensory evoked events (Garaschuk et al., 2000; Minlebaev et al., 2011; J.-W. Yang et al., 2012). While cINs are recruited by these activities (Cossart, 2011; Le Magueresse and Monyer, 2013), whether this influences SST cIN development has not been fully established. To address the impact of activity on these cINs, we chose to selectively and cell-autonomously dampen or augment their excitability during the first few weeks of development. This represents a perinatal period in cIN development during nascent circuit formation, where they are robustly forming or losing synaptic contacts (Allene et al., 2008; Minlebaev et al., 2011; J.-W. Yang et al., 2012; J. W. Yang et al., 2009). SST cINs in the primary somatosensory cortex (S1) were targeted using AAV viral injections in mice carrying an SSTCre allele and a conditional compound synaptophysin1-eGFP (Syp-eGFP) construct, which functions as a presynaptic reporter (i.e SSTre;R26R LSL-tTa;Tg-TRE::Syp-eGFP, Figure1A) (Basaldella et al., 2015; Li et al., 2010; Wamsley et al., 2018). To modulate the activity of SST cINs, these mice were injected at PO with Cre-dependent AAVs that drive the expression of either KIR2.1 or NaChBac channels along with an mCherry reporter (Figure 1A, B). Both channels are voltage-sensitive and have proven to be useful tools to manipulate cellular excitability. The KIR2.1 channel is an inward rectifying potassium channel, which upon overexpression lowers the resting membrane potential towards the reversal potential of $\mathrm{K}^{+}(\sim 90 \mathrm{mV})$ (Bortone and Polleux, 2009; De Marco García et al., 2011; Karayannis et al., 2012; Priya et al., 2018; Yu et al., 2004), thus reducing neuronal excitability. The $\mathrm{NaChBac}$ channel has an activation threshold that is $15 \mathrm{mV}$ more negative than endogenous voltage-gated $\mathrm{Na}^{+}$channels and remains open for 10 times longer (Lin et al., 2010) and therefore augments excitability. To assess the development of the synaptic efferents of infected SST cINs, we allowed pups to mature until juvenile age (P21). Mice were then sacrificed and underwent immunohistochemistry (IHC) to visualize mCherry, Syp-eGFP and gephyrin, followed by confocal 
imaging and axonal and puncta analysis (Ippolito and Eroglu, 2010). We quantified the density of axon arbors of SST cINs by assessing the number of the mCherry-labeled axons restricted to L1 (Figure 1C). We found that the expression of KIR2.1 in SST cINs significantly reduced axonal density and arborization (141.71 \pm 90.45 control (ctl) axon/um² vs 39.65 $\pm 13.63 \mathrm{KIR} 2.1$ axon/um², pVal= <0.00001) whereas the expression of $\mathrm{NaChBac}$ increased the axonal density in $\mathrm{L} 1$ compared to controls

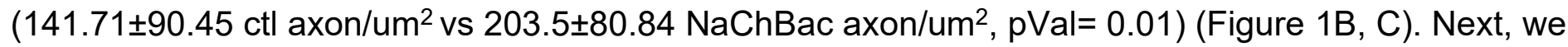
quantified SST synapse density identified through the colocalization of the virally mediated mCherry reporter, Syp-eGFP and the postsynaptic marker gephyrin (mCherry+/GFP+/gephyrin+ puncta), as a proxy for synaptic contacts (Figure 1D, Ippolito and Eroglu, 2010). In SST cINs, KIR2.1 expression resulted in a significant reduction of L1 SST cIN efferent synaptic puncta in comparison to control cells (0.109 \pm 0.009 puncta/um ${ }^{2}$ ctl vs 0.049 \pm 0.006 puncta/um² KIR2.1, pVal= 0.0001) (Figure 1E). By contrast, the overexpression of NaChBac within SST cINs resulted in a robust increase in L1 synaptic puncta $\left(0.109 \pm 0.009\right.$ puncta/um ${ }^{2}$ ctl vs $0.218 \pm 0.030$ puncta/um ${ }^{2}$ NaChBac, pVal= 0.008) (Figure 1D, E). These results suggest that activity has a profound effect on the density of SST cIN axons and synapses. Dampening excitability decreases the number of efferent synaptic structures and axonal arbors of SST cINs, while augmenting it increases both.

\section{Neuronal activity influences alternative splicing and Nova expression within SST cINs}

A growing number of studies indicate that activity-dependent alterative splicing (AS) contributes to the regulation of gene expression and the fine-tuning of transcriptional programs related to synaptic refinement (Eom et al., 2013; lijima et al., 2011b; Mauger et al., 2016; Quesnel-Vallières et al., 2016; Vuong et al., 2016). This prompted us to test whether neuronal activity itself changes the level of AS within SST cINs during circuit formation. To do so, we used electro-convulsive shock (ECS) during peak synaptogenesis (P8) in SSTCre;Ai9 mice. The ECS method generates an acute and reproducible increase in neuronal activity in vivo (Guo et al., 2011; Ma et al., 2009), resulting in increased expression of immediate early genes (IEG) such as, Fos, Egr1, Npas4 and Arc (Figure S1A-E), analogous to that 
observed with $\mathrm{KCl}$ treatment in vitro but with the added advantages of being in vivo and transient. Two to three hours following ECS, we isolated SST cINs from the S1 cortex of SSTre;Ai9 animals using fluorescence activated cell sorting (FACS) (Figure 2A). Sorted SST cINs were used to prepare cDNA libraries that were subsequently sequenced in order to investigate changes in AS (spliced exon: SE, mutually exclusive exons: MXE, retained intron: RI, alternative 5' splice site: A5, alternative 3' splice site: A3) (Figure2A, right). We found 312 transcripts differentially spliced between sham and ECS (FDR $<0.05,|\Delta \psi| \geq 0.1$ threshold), comprised by 139 SE events (82 included and 57 excluded exons), $66 \mathrm{RI}$ events (53 included and 13 excluded introns), $31 \mathrm{MXE}$ events (26 included and 29 excluded exons), 13 A5 events (12 included and 1 excluded exons), and 39 A3 (16 included and 23 excluded exons) (Figure 2B).

Utilizing the SST cIN transcriptome as a reference, we performed gene ontology (GO) analysis to ask if the genes subject to differential AS were enriched for specific functional categories within these neurons. GO analysis of the genes that underwent activity-dependent AS belong to specific ontological categories, such as synapse maturation, synaptic transmission, and axonal growth (Figure 2E). For example, we observed and validated (Table S3) that within activity stimulated SST cINs the Nrxn1 mRNAs exclude exon 10. Notably, this exon lies within a laminin-protein coding domain important for the cell adhesion properties of Nrxn1 at the synapse (Figure 2C, sham, grey vs ECS, green). We next asked whether the activity-dependent AS genes formed a protein-protein interacting network (PPI) based on previously established direct protein interactions in vivo (Rossin et al., 2011). Notably, the genes subjected to activity-dependent AS within SST cINs form highly connected networks illustrating they likely function together to support pre-synaptic vesicle function (Figure 2F, pink), post-synaptic organization and receptor-associated synaptic components (Figure 2F, blue) (pVal<0.0009, 1000 permutations). These genes among others include: Hspa8, Nrxn1, Syngap1, Cacna1c, Ppp3ca, and Grin1 (Figure 2F). These results indicate that augmenting activity within SST cINs during nascent circuit development robustly increases AS events and a majority of the spliced mRNAs are genes specifically related to axonal development and synaptic transmission (Figure2F, pink and blue, respectively). 
We next sought to identify RNA binding proteins (RNABPs) that could mediate activitydependent AS events within SST cINs. To do so, we utilized the RNAseq experiments described above (sham vs. ECS) to perform a motif enrichment analysis that utilizes position probability matrices of binding motifs from 102 RNABPs (i.e. PTBP1/2, FUS, ELAVL4, SRRM4, Rbfox1, FMR1, Nova1, Nova2) (Liu et al., 2017; Park et al., 2016; Y. Yang et al., 2016). Previous HITS-CLIP analysis has revealed that Nova1 and Nova2 share an almost identical RNA-binding domain (YCAY) (Licatalosi et al., 2008; Ule et al., 2006; 2003b; Yuan et al., 2018a). Strikingly, the Nova-binding motif was found to be significantly enriched within activity-dependent targets and at a higher frequency than other neuronal splice factors (i.e. Sam68 (KHDRBS1), SLM2 (KHDRBS2), and Rbfox1) (pVal<0.0001) (Figure 2D). This finding implicates Nova proteins as playing a fundamental role in directing SST cIN activitydependent AS.

\section{Neuronal activity during cortical development influences the expression and localization of}

\section{Nova proteins in SST cINs}

We next examined the expression of Nova1 and Nova2 within SST cINs across development and whether their expression is affected by changes in neuronal activity. Utilizing IHC and genetic fate mapping, we observed that the expression of the Nova family (Nova1 and Nova2) of splice regulators begins within cIN populations soon after they become postmitotic and expressed in $100 \%$ of SST and PV cINs by adulthood (Figure S2A, B). For comparison we also examined Nova expression in excitatory cells (Fig 3B) and 5HT3aR clNs (Figure S2B, C) within this same region. To specifically examine the expression of Nova1 and Nova2 during SST cIN synaptogenesis, we performed quantitative-PCR (qPCR) on FACS isolated cINs from the S1 cortex of Tg-Lhx6::eGFP mice at P2, P8, and P15. The TgLhx6::eGFP mice express eGFP in both SST and Parvalbumin (PV) cINs soon after they become postmitotic. We found that both Nova1 and Nova2 are expressed within all SST and PV cINs across the first two weeks of postnatal development, coinciding with nascent circuit development (Figure 3A, Figure S2B). Interestingly, at P2 the expression of both Nova1 and Nova2 in cINs is significantly higher 
than in cortical excitatory neurons (cExt, Figure 3B). Taken together, we find that both Nova1 and

Nova2 splice factors are highly expressed highly in all SST cINs during circuit integration and may therefore control integral aspects of their development through activity-dependent alternative splicing.

We next investigated whether Nova1 and Nova2 are activity-regulated within SST cINs by examining both their expression and localization during the peak of nascent circuit integration. To do so, we subjected $S S T^{C r e} ; A i 9$ mice to ECS during synaptogenesis (Figure 3C). Next, we performed qPCR for Nova1 and Nova2 within FAC sorted clNs from S1 cortex of either sham or ECS-treated animals. Following 1.5 hours post seizure-induction (1.5 HRPS), we found that the mRNA expression levels of both Nova1 and Nova2 were increased in ECS-treated SST cINs compared to controls (Nova1: 28.4 \pm 5.59 ECS vs 7.61 \pm 0.25 sham; Nova2 pVal=0.002: $10.32 \pm 1.80$ ECS vs $6.59 \pm 0.28$ sham pVal=0.005, Figure 3D). Next, we probed Nova1 and Nova2 protein levels using western blot of sorted SST cINs from S1 cortex. Consistent with an activity-mediated upregulation in Nova expression, we found a significant increase in both Nova1 and Nova2 protein levels $(0.824 \pm 0.0412$ pixel density Nova1 sham vs $5.62 \pm 0.969$ pixel density Nova1 2HRPS, pVal= 0.038 and $0.997 \pm 0.409$ pixel density Nova2 sham vs $5.7 \pm 0.582$ pixel density Nova2 2 HRPS, pVal= 0.022 , pixel densities normalized to $ß$-Actin) (Figure 3E-F). Thus, these results confirm an increase in Nova mRNA and protein expression in SST clNs following an acute increase in neuronal activity.

Following seizure activity Nova proteins have been shown to translocate into the nucleus within excitatory neurons (Eom et al., 2013). We next sought to explore whether manipulating activity also influences Nova intracellular localization in SST cINs (Figure 3G-K). We hypothesized that an activitymediated increase would direct Nova proteins to the nucleus. We therefore analyzed the ratio of SST cINs with Nova expression within the nucleus versus the cytoplasm following ECS (Figure 3G-I) or after constitutive activity-modulation across the first postnatal month (DIO:AAV injections of KIR2.1 or NaChBac into SSTcre animals at P0) (Figure 3G,J-L). First, we examined Nova localization using IHC 2HRPS following ECS within the S1 cortex of P8 SSTCre;Ai9 mice (Figure 3G-H). We quantified the proportions of SST cINs that express Nova proteins most prominently within the nucleus versus the 
cytoplasm by taking multi-Z-stack images of SST clNs and utilizing DAPI to demark the nuclear boundary. We found that Nova localization was observed in three basic patterns in SST cINs: restricted to the nucleus, cytoplasm restricted, or a combination of both nuclear and cytoplasmic expression (Figure 3G). At P8, in the majority of SST cINs, Nova is either restricted to the cytoplasm or expressed in both the nucleus and cytoplasm (Figure 3I). Following an acute increase in activity (ECS), we found a significant increase in the ratio of nuclear to cytoplasmic Nova protein within SST cINs $(0.948 \pm 0.055$ ratio in sham versus $3.65 \pm 0.465$ ratio in $\mathrm{ECS}, \mathrm{pVal}=0.001$, Figure $3 \mathrm{H}, \mathrm{I})$. Next, by utilizing the same analytical approach, we examined Nova protein localization in S1 of P21 mice that express either KIR2.1 or NaChBac along with an mCherry reporter (Figure 3J, K). We found a substantial increase in the ratio of SST cINs that localized Nova protein in the nucleus compared to the cytoplasm in NaChBacexpressing compared to control cells $(4.75 \pm 0.678$ ratio control vs $40.91 \pm 8.41$ ratio NaChBac, pVal= 0.0004) (Figure 3K, left). In contrast, we found a robust decrease in the ratio of nuclear to cytoplasmic Nova protein within SST cINs injected with KIR2.1 (4.75 \pm 0.678 ratio control vs $0.265 \pm 0.104$ ratio KIR2.1, pVal=<0.0001) (Figure 3K, right). Most striking, we also observed more than half of SST clNs subjected to KIR2.1 expression have substantially reduced levels of Nova protein expression (Figure $3 \mathrm{~L}-\mathrm{N}, \mathrm{pVal}=0.0303)$. Altogether these data indicate that during synaptogenesis Nova protein expression and localization within SST cINs is strongly modulated by acute or persistent changes in activity.

\section{Nova1 and Nova2 control distinct AS networks within SST cINs}

To address whether Nova1 and Nova2 differentially affect connectivity and maturation, we asked what AS networks they control within SST cINs during development. Given that they share a very similar RNA-binding motif and are found associated with one another in vivo, they were thought to function cooperatively (Licatalosi et al., 2008; Racca et al., 2010; Yuan et al., 2018b). However recently, it has been shown that in addition to their synergistic roles, Nova1 and Nova2 proteins each control distinct 
AS gene networks (Saito et al., 2019; 2016). We thus chose to examine changes in AS within SST cINs in Nova1, Nova2 or compound conditional knockout mice (Saito et al., 2019; Yuan et al., 2018b). Using FAC sorting, we isolated SST cINs from SSTCre;Nova1F/F or SSTCre; ;Nova2F/F or SSTCre;Nova1F/F; Nova2F/F mice on an Ai9 reporter background (referred to henceforth at SST-Nova1, SST-Nova2 and SST-dKO, respectively) at P8 (Figure S3A). We prepared cDNA libraries from FAC sorted SST cINs, performed RNA sequencing and assessed AS changes between control SST cINs versus each of these mutant alleles. Compared to wild type controls, Nova1 loss resulted in 124 altered AS events (43 included and 81 excluded), Nova2 loss lead to 339 altered AS events (122 included and 217 excluded) and double mutants exhibited 270 altered AS events (108 included and 162 excluded) (FDR < 0.05$)$ (Figure S3B). These results illustrate that within SST cINs, the loss of Nova2 results in the largest number of changes in mRNA splicing events compared to compound loss of either Nova1 or both Nova genes. We next assessed the overlap of changes in AS events observed within each mutant (Figure S3C-E). We found the number of alterations in SST-Nova2 AS events that overlap with SST-dKO is almost three times higher than that observed when comparing the overlap between SST-dKO and SSTNova1 (i.e. 62 altered SST-Nova2 AS events coincided with the 162 observed in SST-dKO versus an overlap of only 25 AS events that were altered in SST-Nova1 mutants, Figure S3DE). By contrast, less than $15 \%$ of the altered SST-Nova1 AS genes overlap with changes observed in SST-Nova2 mutants (i.e. only 28 of the 217 SST-Nova2 events were altered in SST-Nova1 mutants) (Figure S3C, D). Interestingly, SST-dKO mutants exhibited less altered splicing events than the single SST-Nova2 mutant, suggesting that some inclusion and exclusion AS events are antagonistically directed by Nova1 and Nova2.

To infer their specific biological functions, we performed GO analysis on the altered AS events from each mutant and then asked whether the affected AS events form direct PPI networks. GO analysis of the SST-Nova1 targets did not result in any significant enrichment of specific functional categories (below an FDR of 0.05 ) however it did organize genes into categories such as RNA binding, ion binding, and catalytic activity (Figure S3F). SST-Nova1 AS genes formed a relatively indistinct small 
sparse PPI network (pVal<0.09) representing vesicle-transport and nucleic-acid binding pathways

(Figure S3I, pink shaded). In contrast, SST-Nova2 and SST-dKO AS genes organized into several shared significant GO categories such as neuron projection, axon, cell-cell junction, and synaptic function (FDR <0.05) (Figure S3G, H). SST-dKO AS genes also organized into some unique categories, which were involved in postsynaptic specialization, dendrite, and synaptic vesicle membrane (FDR $<0.05)$ (Figure S3H). We next asked if the AS genes affected in SST-Nova2 and SST-dKO were predicted to function together in a PPI network representing specific biological processes (Figure S3IK). Perhaps not surprisingly both formed highly connected significant PPI networks ( $p$ Val $<0.0009$, 1000 permutations) representing multiple pathways for vesicle-transport, pre- and post-synaptic function and organization, as well as $\mathrm{Ca}^{2+}$ signaling (Figure S3J, K, pink and green respectively). Interestingly, the PPI network for SST-Nova2 uniquely includes numerous glutamate receptors and their adaptors, respectively (i.e. Grin2b, Grik1, Gria3, Grm5 and Grip1, Sharpin, Dlg2) (Figure S3J, highlighted pre-synaptic genes in pink and post-synaptic genes in green). Altogether these results suggest that considering the Nova family as a whole, Nova2 (compared to Nova1) is the main driver of AS and importantly, may be most relevant for synaptic development of SST cINs.

\section{SST-Nova1 and SST-Nova2 mutants have impaired afferent and efferent connectivity.}

To confirm our predictions from the AS analysis of conditional Nova mutants, we next sought to determine the effect of the loss of Nova1 and Nova2 on SST cIN synaptic development and function. To this end, we assessed the requirement for Nova1 and/or Nova2 for both the anatomical connectivity and physiological properties of SST cINs. SST-dKO mice were small in size and while generated at Mendelian ratios, many died as early as P8 and often exhibited seizures (Figure S4A and results not shown). In the single cKO mutants, we used IHC to quantify the density of SST cIN efferent synapses, defined as the apposition of VGAT+ (vesicle GABA transporter) and gephyrin+ puncta from a SST cIN axon within L1 of the S1 cortex at P8 (Figure 4A, black asterisks mark example puncta). We found that both SST-Nova1 (0.281 \pm 0.041 puncta/um² SST-Nova1 vs $0.454 \pm 0.037$ puncta/um ${ }^{2}$ ctl, pVal=0.003) 
and SST-Nova2 (0.197 \pm 0.016 puncta/um ${ }^{2}$ SST-Nova2 vs $0.454 \pm 0.037$ puncta/um ${ }^{2}$ ctl, pVal=<0.0001) exhibited a significant reduction in SST+ synapses compared to control SST synapses within L1 (Figure 4B). To confirm the synaptic phenotype observed, we recorded the inhibitory outputs from SST cINs onto pyramidal cells in L2/3 and L5 using a conditional channelrhodopsin mouse line (Ai32F/F) crossed with SST-Nova1, SST-Nova2 or SST-control mice (Figure 4C). We observed a significant reduction in

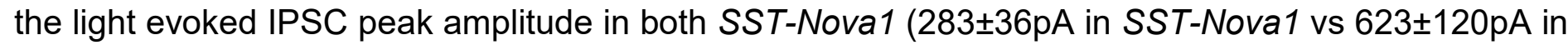
ctl, pVal=0.0037), as well as SST-Nova2 (340 $\pm 85 p A$ in SST-Nova2 vs 766 $\pm 211 \mathrm{pA}$ ctl, pVal=0.0021), confirming that the anatomically observed reduction in synaptic output density is functionally significant in both mutants (Figure 4D SST-Nova1 top orange trace, SST-Nova2 pink trace bottom). While both SST-Nova1 and SST-Nova2 leads to a reduction in both the density of synaptic efferents and their inhibitory function, loss of Nova2 has a bigger impact on efferent connectivity within SST cINs than Nova1 (one-way ANOVA, pVal=0.0001).

We also investigated whether the density of excitatory synapses onto SST cINs is affected by the loss of Nova1 or Nova2. We performed IHC for Vglut1 (vesicular glutamate transporter) and Homer1c on SST-Nova1 and SST-Nova2 dendrites within the S1 cortex at P8 (Figure 4E, black asterisks mark example puncta). We quantified the density of putative excitatory synapses by the overlap of Vglut1+ and Homer1c+ puncta onto mCherry+ dendrites of SST cINs. We found that the number of putative excitatory afferent synapses onto SST-Nova1 and SST-Nova2 is significantly reduced compared to control SST cINs $\left(0.144 \pm 0.016\right.$ puncta/um ${ }^{2}$ SST-Nova1 vs $0.207 \pm 0.022$ puncta/um ${ }^{2}$ ctl, pVal $=0.028$ and $0.137 \pm 0.013$ puncta/um ${ }^{2}$ SST-Nova2 vs $0.207 \pm 0.022$ puncta/um ${ }^{2}$ ctl, pVal= 0.012) $($ Figure 4F). To examine whether these anatomical abnormalities observed in SST-Nova1 and SST-Nova2 mutants affected synaptic function, we performed whole-cell patch clamp recordings to measure miniature excitatory postsynaptic currents (mEPSCs) within SST clNs (Figure 4G). In accordance with the puncta analysis, both SST-Nova1 and SST-Nova2 exhibited significant reductions in the mEPSC frequency (SST-Nova1: $1.16 \pm 0.08 \mathrm{~Hz}$ vs SST-Nova2: $0.39 \pm 0.05 \mathrm{~Hz}$ vs ctl: $2.43 \pm 0.2 \mathrm{~Hz}$, pVal=0.0025, Figure 4H). In addition, we observed a significantly increased mEPSC amplitude in SST- 
Nova2 (SST-Nova2: $-40 \pm 15.7 \mathrm{pA}$ vs SST-Nova1: $-30.12 \pm 13.15 \mathrm{pA}$ vs ctl: $-30.36 \pm 13.34 \mathrm{pA}$, pVal=0.0.0001, Figure S4D-G). Thus, while SST-Nova2 cINs have a striking reduction in their excitatory inputs, the remaining excitatory synapses are functionally stronger than Nova1 or control cINs. Moreover, the intrinsic properties of both $c K O$ alleles were differentially affected. Specifically, we observed that the rheobase was significantly lower for SST-Nova2 compared with either controls or

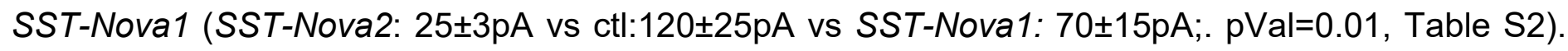

As rheobase is a measurement of the minimum current required to produce an action potential, SSTNova2 clNs are potentially compensating for the loss of excitatory synapses by lowering the minimal current amplitude required for depolarization. Other intrinsic physiological properties are summarized in Table S2. Altogether these results solidify the role of both Nova1 and Nova2 in the synaptic development of SST cINs. Furthermore, consistent with the AS analysis, these results suggest that within SST cINs Nova2 has a larger impact on the changes in synaptic connectivity compared to Nova1.

\section{Nova RNA binding proteins control activity dependent AS in SST cINS during development}

Given that activity increases the expression level and nuclear localization of both Nova proteins, we hypothesized that their loss would result in changes in activity-dependent AS. To this end, we repeated our investigation of how Nova-dependent AS isoforms are altered in mutant mice. This time we examined the changes specifically following ECS within SST cINs during synaptogenesis in vivo. 2-3 hrs following ECS, we isolated SST cINs from SST-dKO mice (Figure 5A). Following augmentation of neuronal activity, we found that the loss of both Nova genes results in the differential splicing of 346 transcripts (FDR $<0.05,|\Delta \psi| \geq 0.1$ ). These are broken down into 166 SE events (106 included and 60 excluded exons), 72 RI events (51 included and 21 excluded introns), 70 MXE events (37 included and 33 excluded exons), 9 A5 events (7 included and 2 excluded exons), and 30 A3 (9 included and 21 excluded exons) (Figure 5B). Independent fluorescent RT-PCR amplifications with primers flanking the alternatively spliced segments confirmed the observed AS changes. We were able to validate $70 \%$ of targets tested (Table S3). For example, we validated the activity-dependent inclusion of exon 14 in 
Syngap1, a gene associated in multiple disorders including epilepsy and important for excitatory postsynaptic function (Figure 5C, D). As predicted from RNAseq, SST cINs subjected to acute increases in activity from SST-dKO animals, compared to control SST cINs, exhibit a significant reduction in the expression of Syngap1 exon 14 (Figure 5D).

We found the majority of genes which undergo activity-induced Nova-dependent differential splicing were significantly enriched for GO categories such as pre-synaptic vesicular function, synapse organization, synaptic transmission, and neuronal growth (Figure 5E). Many of the genes within these categories are known to have important functions for axon organization and synaptogenesis such as, Nrxn1, Nrxn3, PlxnA2, and EphA5. Interestingly, the activity-dependent Nova AS targets were strikingly enriched for excitatory post-synaptic specializations such as, Shank1, Syngap1, Dlg3, Grin1 and Gria1. Furthermore, these genes are predicted to function together in a direct PPI network representing specific pre-synaptic and post-synaptic biological processes (direct network pVal $=0.0009,10000$ permutations, Figure 5F). For example, the loss of Nova leads to an altered activity-dependent splicing program of multiple genes important to NMDA receptor-mediated signaling (Grin1) connected with PSD organization (i.e. Dlg3, Shank1) and $\mathrm{Ca}^{2+}$-dependent signaling (i.e Hras, Rapgef1) (Figure 5F).

In sum, the activity-mediated Nova-dependent AS changes within SST cINs are central for finetuning of synaptic and axonal development. We previously found that another important RNABP, Rbfox1, influences axonal development and also shuttles from the cytoplasm to the nucleus upon increase in activity in SST cINs (Lee et al., 2009; Wamsley et al., 2018). However, upon comparing the activity-dependent splicing programs within SST cINs of Rbfox1 (69 activity-dependent events) to Nova1/2 (346 activity-dependent events), we found Nova proteins control a much larger number of activity-dependent splicing events. This supports our hypothesis that Nova proteins are key players in the control of activity-dependent AS (Figure S5A).

Nova2 overexpression within SST cINs augments axonal growth and synaptic formation in an activity dependent manner 
Activity increases both the expression of Nova proteins as well as synapse formation; while conversely there is a striking decrease in synaptogenesis after loss of Nova function. Moreover, from our analysis of SST cIN cKOs, it was evident that of the two Nova proteins, Nova2 has the more profound effect on the AS of genes involved in synaptogenesis. We therefore examined whether over expression (OE) of Nova2 alone could phenocopy the observed changes in connectivity within SST clNs. To that end, we overexpressed Nova2 specifically in SST+ neurons using an AAV virus (AAVTRE-Nova2-mCherry) together with a Cre-dependent synaptophysin1-eGFP virus (AAV-hSyn-DIOSynaptophysin1-eGFP) in $S S T^{\text {Cre }}: t T A^{F / F}$ mice within the $S 1$ cortex (Figure 6A). Remarkably, the overexpression of Nova2 had a similar effect as increasing activity; the axonal density in L1 of SST clNs was significantly increased compared to controls $\left(118.5 \pm 53\right.$ axon/um ${ }^{2}$ controls vs $198.2 \pm 23$ axon/um²-Nova2 OE; pVal=0.0012) (Figure 6B, C, E). Additionally, this increased axonal density was associated with increased synaptogenesis. We quantified the density of putative synaptic puncta as defined by the juxtaposition of presynaptic Syp-eGFP from the OE Nova2 SST neurons colocalized with gephyrin $\left(0.026 \pm 0.0031\right.$ synaptic puncta/um ${ }^{2}$ in ctl vs $0.055 \pm 0.01$ synaptic puncta/um ${ }^{2}$ in the case of Nova2 OE; pVal=0.04) (Figure 6F, G, I). Moreover, as in the case of increasing activity (either constitutively, NaChBac, or acutely, ECS), the nuclear localization of Nova was robustly increased when Nova2 was overexpressed in the SST cINs (Figure 6J, L).

We next asked whether suppressing activity would affect the increased axogenesis and synaptogenesis observed with the Nova2 OE. We expressed KIR2.1 in SST cINs as described earlier, together with the TRE-Nova2-mCherry in the S1 cortex (Figure 6A). The dual expression of Nova2 OE and KIR2.1 within SST cINs prevented the increased axogenesis that was observed with Nova2 OE alone $\left(198 \pm 23\right.$ axon/um ${ }^{2}$ in Nova2 OE vs $30.37 \pm 10.51$ axon/um ${ }^{2}$ in the case of Nova2 OE\&KIR2.1, pVal=0.0053) (Figure 6D, E). As observed with KIR2.1 alone, this decreased axogenesis also corresponded with decreased synaptogenesis $\left(0.055 \pm 0.011\right.$ synaptic puncta/um ${ }^{2}$ in Nova2 OE vs 0.024 \pm 0.007 synaptic puncta/um ${ }^{2}$ Nova2OE+KIR2.1, pVal=0.0042) (Figure 6H, I). Additionally, the increased nuclear localization of Nova that was observed with the Nova2 OE was abolished when the 
activity of the cells was cell-autonomously reduced using KIR2.1 (Figure 6K, L). Perhaps most strikingly, as with our initial KIR2.1 experiment, the levels of Nova2 protein despite being constitutively OE were strongly reduced in cells co-expressing KIR2.1 (Figure 6K). This provides strong evidence that the stability of Nova protein is dependent on the level of basal activity within SST cINs.

\section{Discussion}

In the present study, we have examined the effect that both neuronal activity and the Nova RNABPs have on the synaptogenesis of SST cINs. Our analysis began with the observation that activity levels strongly influence the maturation of SST cINs. Acutely evoking activity during circuit integration with ECS resulted in both transcriptional and translational upregulation of Nova proteins. This was accompanied by a striking change in AS of synaptic genes and synaptogenesis within SST cINs. We then systematically examined whether a causal relationship exists between these three observations.

Our results indicate that within this interneuron population, particularly during periods of circuit maturation, excitation levels correlate with changes in AS and the formation of afferent/efferent connectivity. These events appear to be tightly linked to Nova function, as the expression, localization and splicing activity of both Nova1 and Nova2 proteins are strongly modulated by activity. Examination of how splicing events are impacted by Nova single and compound cKOs in SST cINs demonstrate that developmental RNA splicing events in these cells are particularly impacted by the loss of Nova2. This is mirrored by the magnitude in reduction of excitatory input and inhibitory output within SST cINs, accompanied by a structural decrease in their synaptic contacts. Conversely, over-expression of Nova2 in SST cINs greatly enhances synaptogenesis, a phenomenon that can be suppressed by simultaneous dampening of excitability. Together these findings demonstrate that activity is coupled to synaptogenesis by a mechanism involving Nova-mediated splicing of synaptic proteins.

We and others have shown that activity regulates programmed cell death Priya:2018hc, Marin:2016bi, Denaxa:2018iu, Wong:2018fu\}. However, we observed no indication that the loss of Nova2 impacted SST cIN survival. In addition, we observed that NaChBac and KIR2.1 could modulate 
synaptogenesis in SST cINs both during and after the peak of cell death in this region (data not shown).

Conversely, the number of phenotypic changes observed in conditional Nova loss of function mutants suggests that these genes have effects beyond synaptogenesis. Nova2, in particular, also targets genes involved in protein trafficking to the membrane, cell-cell signaling, and neurotransmitter/ion channel function, indicating it influences multiple aspects of SST cIN maturation. In addition, prior work from the Darnell lab has demonstrated a role for Nova2 in both migration and axonal pathfinding within the cortex, spinal cord and brain stem (Saito et al., 2016; Yano et al., 2010). Taken together clearly much remains to be understood concerning the role Nova proteins play during development in specific brain regions, circuits, and cell types.

While Nova function is a core regulator of alternative splicing in SST cINs, it represents only one of a host of RNABPs utilized both within this cell type, as well as across neurons in general. A recent study demonstrated that within the mature CNS many classes of neurons, including SST cINs, can be classified both by their expression levels of RNABPs and their corresponding repertoire of alternatively spliced mRNAs (Furlanis et al., 2019). Comparison of their work to our present findings illustrate that both the expression of RNABPs and the patterns of AS are strongly regulated across development and are responsive to changes in neuronal activity. RNA binding splice factors have previously been shown to promote alternative splicing of synaptic proteins in response to neuronal depolarization and $\mathrm{Ca}^{2+}$ signaling (Eom et al., 2013; Mauger et al., 2016; Quesnel-Vallières et al., 2016; Vuong et al., 2016), For example, previous research demonstrated that the splicing of neurexins, a gene family known to function in synaptogenesis, are mediated through the actions of the SAM68 splicing factor (lijima et al., 2011b). Similarly, It has also been illustrated that neuronal activity reduces the expression of the SRRM4 RNA-binding protein, which resulted in altered RNA splicing and a corresponding decrease in excitatory synapses (Quesnel-Vallières et al., 2016). As such AS represents a largely unexplored but central genetic mechanism, capable of directing cell-type specific development and specification.

Understanding both the repertoire of splice factors and the cell-specific patterns of splicing across development will undoubtedly provide further insight into how AS influences cIN development. 
One could imagine systematically examining the role of these differential splice mRNA variants through combinatorial knockdown or over-expression. However, this would be technically challenging, even if restricted to only those that are Nova-dependent. As we show here many of these AS genes have been shown to function together (PPI networks), knocking down one or a few would most likely result in the same phenotype. In addition, the abundance of the specific splice forms of different genes within SST clNs is relative rather than absolute. Such that the identity, amount, and function of the genes that make up a cell's alternatively spliced gene network will change dependent on cell identity and state (i.e. developmental period, neuronal activity, etc). Further complicating matters, activity also affects the overall transcription level of these same genes. As a result, the abundance of particular splice forms co-vary as a function of both transcription and AS. Taken together, this argues that conditional removal of RNABPs, such as Nova2, is a more biologically relevant approach to understanding the role of AS within discrete cell types.

Our results show a clear interdependence between activity, Nova function and synaptogenesis in SST clNs. The dual observations that 1) Nova2 over-expression is sufficient to increase synaptogenesis and 2) that co-expression of KIR2.1 can suppress this effect, demonstrate that the two are functionally coupled. Most obviously this appears to be regulated through an activity-dependent increase in Nova expression (see model Figure 6M). When SST cIN activity is increased with ECS or with NaChBAC expression, Nova transcripts as well as protein are upregulated and shuttled to the nucleus. The mechanisms for activity-dependent changes in Nova expression and localization are unknown. It is possible that the Nova gene loci may contain binding sites for immediate-early-genes (i.e. cFOS, Jak/Jun, EGF) or specific activity-dependent transcription factors (i.e. NPAS4, Satb1). With regard to control of its localization, previous work has discovered a nuclear-localization signal (NLS) within the Nova protein domains. It is however unknown whether their activation is mobilized by splicing or post-translational modifications. For instance, Rbfox 1 undergoes activity-dependent mRNA splicing that results in exposure of an NLS and localization to the nucleus (Lee et al., 2009; Wamsley et al., 2018). Furthermore, our results indicate that activity itself regulates Nova2 RNA and protein stability. 
In the presence of KIR2.1, the levels of Nova protein appear to be dramatically reduced, even when Nova2 is over-expressed. In this latter context, clearly Nova2 levels are not constrained by mRNA production. These results indicate that the stability of Nova protein is at least partly dependent on activity. Taken together, these findings indicate that there exist multiple mechanisms by which cell activity is coupled to Nova function and AS within SST cINs. Given its broad expression and the strong phenotypes associated with both conditional and global Nova loss of function, studies of this RNABP will no doubt provide further insights into their contribution to normal and disease brain function. 


\section{CONTACT FOR REAGENT AND RESOURCE SHARING}

Please contact GF for reagents and resources generated in this study.

\section{EXPERIMENTAL MODEL AND SUBJECT DETAILS}

\section{- Mouse maintenance and mouse strains}

All experimental procedures were conducted in accordance with the National Institutes of Health guidelines and were approved by the Institutional Animal Care and Use Committee of the NYU School of Medicine and Harvard Medical School. Generation and genotyping of, SSTCre (Taniguchi et al., 2011), RCE ${ }^{\mathrm{eGFP}}$ (Sousa et al., 2009), Lhx6 BAC transgenic (referred to as tgLhx6;eGFP) (Gong et al., 2003), Nova1LoxP/LoxP(Yuan et al., 2018a), Nova2LoxP/Lox(Saito et al., 2019), TRE-Bi-SypGFP-TdTomato (Li et al., 2010), and Ai9 LoxP/LoxP Ai32 LoxP/LoxP, Rosa-tTa LoxP/LoxP.(commercially available from Jax laborites). All mouse strains were maintained on a mixed background (Swiss Webster and C57/ B16). The day of birth is considered P0. Information about the mouse strains including genotyping protocols can be found at http://www.jax.org/ and elsewhere (see above references).

\section{METHOD DETAILS}

\section{- Immunochemistry and imaging}

Embryos, neonate, juvenile and adult mice were perfused inter cardiac with ice cold $4 \%$ PFA after being anesthetized on ice (neonates) or using Sleepaway injection. Brains that were processed for immunofluorescence on slides were post-fixed and cryopreserved in $30 \%$ sucrose. $16 \mu \mathrm{m}$ coronal sections were obtained using Cryostat (Leica Biosystems) and collected on super-frost coated slides, then allowed to dry and stored at $-20^{\circ} \mathrm{C}$ until use. For immunofluorescence, cryosections were thawed and allowed to dry for 5-10 min and rinsed in 1x PBS. They were incubated at room temperature in a blocking solution of PBST (PBS-0.1\%Tx-100) and 10\% normal donkey serum (NDS) for $1 \mathrm{hr}$, followed by incubation with primary antibodies in PBS-T and $1 \%$ NDS at $4^{\circ} \mathrm{C}$ overnight or 2 days. Samples were then washed 4 times with PBS-T and incubated with fluorescence conjugated secondary Alexa antibodies (Life Technologies) in PBS-T with 1\% NDS at room temperature for $1 \mathrm{hr}$. Slides were incubated for $5 \mathrm{~min}$ with DAPI, washed 3 times with PBS-T. Then slides were mounted with Fluoromount 
G (Southern Biotech) and imaged.

Brains that were processed for free-floating immunofluorescence were first post-fixed in $4 \%$ PFA overnight at $4^{\circ} \mathrm{C} .50 \mu \mathrm{m}$-thickness brain slices were taken on a Leica vibratome and stored in a cryoprotecting solution (40\% PBS, 30\% glycerol and 30\% ethylene glycol) at $-20^{\circ} \mathrm{C}$. For immunofluorescence, floating sections were blocked for $1 \mathrm{hr}$ at RT in normal donkey or goat serum blocking buffer and incubated for $2-3$ days at $4^{\circ} \mathrm{C}$ with primary antibodies in blocking buffer. Sections were washed $4 \times 30$ min at RT in PBST, incubated overnight at $4^{\circ} \mathrm{C}$ with secondary antibodies and DAPI in blocking buffer, washed $4 \times 30$ min at RT in PBST before being mounted on super-frost plus glass slides. Primary antibodies are listed in Key Resource Table.

\section{- Nova1/2 Localization}

To quantify the Nova localization in SST cINs, mCherry+/SST cIN or KIR2.1+/SST cINs or $\mathrm{NaChBac+/SST}$ cIN or Sham+/SST cIN or ECS+/SST cINs or Nova2OE/SST cIN or Nova2OE+KIR2.1/SST cINs were binned into two categories based on the cell compartment Nova1/2 protein was localized to: Cytoplasmic restricted or Nuclear-expressing (comprised of nuclear restricted or whole soma localization). The number of Nuclear-expressing cells was then divided by the number of cytoplasmic restricted cells to obtain a ratio for Nova localization from either mCherry+/SST cIN or KIR2.1+/SST cINs. This was collected from at least three tissue sections from at least 3 animals.

\section{- Electroconvulsive Shock}

Electroconvulsive stimulation (ECS) was administered to animals with pulses consisting of $1.0 \mathrm{~s}, 50$ $\mathrm{Hz}, 75 \mathrm{~mA}$ stimulus of $0.7 \mathrm{~ms}$ delivered using the Ugo Basile ECT unit (Model 57800, as previously described (Guo et al., 2011; Ma et al., 2009). Sham animals were similarly handled the exact same procedure but without the current administration.

\section{- Confocal imaging and synaptic puncta analysis}


Animals were perfused as described above. Post-fixation incubation prior to cryopreservation was skipped. Cryostat sections $(16 \mu \mathrm{m})$ were subjected to IHC as described above. Images were taken within the S1 cortex of at least three different sections from at least three different animals per genotype with a Zeiss LSM 800 laser scanning confocal microscope. Scans were performed to obtain 4 optical Z-sections of $0.33 \mu \mathrm{m}$ each (totaling $\sim 1.2 \mu \mathrm{m}$ max projection) with a $63 \mathrm{x} / 1.4$ Oil DIC objective. The same scanning parameters (i.e. pinhole diameter, laser power/offset, speed/averaging) were used for all images. Maximum projections of 4 consecutive $0.33 \mu \mathrm{m}$ stacks were analyzed with ImageJ (NIH) puncta analyzer plugin (Ippolito and Eroglu, 2010) to count the number of individual puncta consisting of presynaptic and post-synaptic markers that are close enough together to be considered a putative synaptic puncta. Synaptic puncta density per image was calculated by normalization to total puncta acquired for each individual channel accounted in each image for each condition. Puncta Analyzer plugin is written by Barry Wark, and is available for download (https://github.com/physion/puncta-analyzer). Nova protein intensity was performed as:Cryostat sections of 20 um were immunostained with goat antimCherry and human anti-pan Nova (from Darnell Lab). Images were analyzed using Fiji/ImageJ and Nova1/2 protein intensity levels were assessed normalized against area of the cells expressing the AAV.

\section{- Electrophysiological recordings}

Slice preparation: Acute brain slices (300 $\mu \mathrm{m}$ thick) were prepared from P18-P22 mice. Mice were deeply anesthetized with isofluorane. The brain was removed and placed in ice-cold modified artificial cerebrospinal fluid (ACSF) of the following composition (in mM): $87 \mathrm{NaCl}, 26 \mathrm{NaHCO}, 2.5 \mathrm{KCl}, 1.25$ $\mathrm{NaH} 2 \mathrm{PO} 4,0.5 \mathrm{CaCl}, 4 \mathrm{MgCl}$, 10 glucose, 75 sucrose saturated with $95 \%$ O2, 5\% CO2 at $\mathrm{pH}=7.4$. Coronal sections were cut using a vibratome (Leica, VT 1200S). Slices were then incubated at 34C for 30 minutes and then stored at room temperature until use.

Recordings: Slices were transferred to the recording chamber of an up-right microscope (Zeiss Axioskop) equipped with IR DIC. Cells were visualized using a 40X IR water immersion objective. Slices were perfused with ACSF of the following composition (in mM): $125 \mathrm{NaCl}, 25 \mathrm{NaHCO}, 2.5 \mathrm{KCl}, 1.25$ 
$\mathrm{NaH} 2 \mathrm{PO} 4,2 \mathrm{CaCl}$, $1 \mathrm{MgCl} 2,20$ glucose, saturated with 95\% O2, 5\% CO2 at pH=7.4 and maintained at a constant temperature $\left(31^{\circ} \mathrm{C}\right)$ using a heating chamber. Whole-cell recordings were made from randomly selected tdTomato-positive SST interneurons or tdTomato negative pyramidal cells from layer II-III or layer $\mathrm{V}$ of the somatosensory cortex. Recording pipettes were pulled from borosilicate glass capillaries (Harvard Apparatus) and had a resistance of 3-5 $\mathrm{M} \Omega$ when filled with the appropriate internal solution, as reported below. Recordings were performed using a Multiclamp 700B amplifier (Molecular Devices). The current clamp signals were filtered at $10 \mathrm{KHz}$ and digitized at $40 \mathrm{kHz}$ using a Digidata 1550A and the Clampex 10 program suite (Molecular Devices). Miniature synaptic currents were filtered at $3 \mathrm{kHz}$ and recorded with a sampling rate of $10 \mathrm{kHz}$. Voltage-clamp recordings where performed at a holding potential of $0 \mathrm{mV}$. Current-clamp recordings were performed at a holding potential of $-70 \mathrm{mV}$. Cells were only accepted for analysis if the initial series resistance was less than $40 \mathrm{M} \Omega$ and did not change by more than $20 \%$ throughout the recording period. The series resistance was compensated online by at least $\sim 60 \%$ in voltage-clamp mode. No correction was made for the junction potential between the pipette and the ACSF.

Passive and active membrane properties were recorded in current clamp mode by applying a series of hyperpolarizing and depolarizing current steps and the analysis was done in Clampfit (Molecular Devices). The cell input resistance was calculated from the peak of the voltage response to a $50 \mathrm{pA}$ hyperpolarizing $1 \mathrm{sec}$ long current step according to Ohm's law. Analysis of the action potential properties was done on the first spike observed during a series of depolarizing steps. Threshold was defined as the voltage at the point when the slope first exceeds a value of 20 V.s-1. Rheobase was defined as the amplitude of the first depolarizing current step at which firing was observed. Analysis of miniature inhibitory events was done using Clampfit's template search.

Pipette solutions: Solution for voltage-clamp recordings from pyramidal cells (in mM): 125 Csgluconate, 2 CsCl, 10 HEPES, 1 EGTA, 4 MgATP, 0.3 Na-GTP, 8 Phosphocreatine-Tris, 1 QX-314-Cl and $0.4 \%$ biocytin, equilibrated with $\mathrm{CsOH}$ at $\mathrm{pH}=7.3$. Solution for current clamp recordings from SST 
cINs (in mM): 130 K-Gluconate, $10 \mathrm{KCl}, 10$ HEPES, 0.2 EGTA, 4 MgATP, 0.3 NaGTP, 5

Phosphocreatine and $0.4 \%$ biocytin, equilibrated with $\mathrm{KOH} \mathrm{CO} 2$ to a $\mathrm{pH}=7.3$.

\section{- Nova2 OE/ Nova2 OE +KIR2.1 Experiment}

SSTCre mice crossed with Rosa-tTa LoxP/LoxP. were injected at P0/1 with either AAV2/1-DIO-SypGFP at 1:2 dilution with PBS; or AAV2/1-DIO-SynGFP + TRE-Nova2-mCherry (myc tagged) at 1:1:1 dilution with PBS; or AAV2/1-DIO-SynGFP + TRE-Nova2-mCherry (myc tagged) + AAV2/1-DIO-Kir2.1mCherry at 1:1:1 ratio in the $\mathrm{S} 1$ cortex. Mice were perfused at P21, brains harvested, sucrose protected and sectioned on a freezing microtome (Leica) at 20um thickness as described above. Primary antibodies are listed in Key Resource Table.

\section{- Optogenetic stimulation}

Blue-light $(470 \mathrm{~nm})$ was transmitted to the slice from an LED placed under the condenser of an up-right microscope (Olympus BX50). IPSCs were elicited by applying single 1 ms blue-light pulses of varying intensities (max. stimulation intensity $\sim 0.33 \mathrm{~mW} / \mathrm{mm}^{2}$ ) and directed to layer2/3 or layer5 of the slice in the recording chamber. Light pulses were delivered every 5 seconds. The LED output was driven by a TTL output from the Clampex software of the pCLAMP 9.0 program suite (Molecular Devices).

\section{- Isolation of cortical interneurons from the developing mouse cerebral cortex}

Cortical interneurons were dissociated from postnatal mouse cortices (P8) as described (Wamsley et al., 2018). We collected at least 3-5 cKO and 3-5 ct/ brains and maintained overall balanced numbers of females and males within each condition, in order to avoid sex- related gene expression biases. Following dissociation, cortical neurons in suspension were filtered and GFP+ or TdTomato+ fatemapped interneurons were sorted by fluorescence activated-cell sorting (FACS) on either a Beckman Coulter MoFlo (Cytomation), BD FACSAria II SORP or Sony SY3200. Sorted cINs are collected and lyzed in $200 \mu \mathrm{l} \mathrm{TRIzol}$ LS Reagent, then thoroughly mixed and stored at $-80^{\circ} \mathrm{C}$ until further total RNA extraction. 
- Nucleic acid extraction, RNA amplification, cDNA library preparation and RNA sequencing

Total RNAs from sorted SST cINs (P8 mouse S1 cortices for Figure 2, Figure S3, and Figures 5) were extracted using TRIzol LS Reagent and PicoPure columns (if $<20 \mathrm{~K}$ cells were recovered) or PureLink RNA Mini Kit (if $>20 \mathrm{~K}$ cells were recovered), with PureLink DNase for on-column treatment, following the manufacturers' guidelines. RNA quality and quantity were measured with a Picochip using an Agilent Bioanalyzer and only samples with high quality total RNA were used (RIN: 7-10). 20ng of total RNA was used for cDNA synthesis and amplification, using NuGEN Ovation RNA-Seq System V2 kit (NuGEN part \# 7102). 100 ng of amplified cDNA were used to make a library using the Ovation Ultralow Library System (NuGEN part \# 0330). The samples were mulitplexed and subjected to 50-nucleotide paired-end read rapid with the Illumina HiSeq 2500 sequencer ( $\mathrm{v} 4$ chemistry), to generate $>50$ million reads per sample. Library preparation, quantification, pooling, clustering and sequencing was carried out at the NYULMC Genome Technology Center. qRT-PCR (quantitative RT-PCR) was performed using SYBR select master mix (Thermo-Fisher Scientific) on cDNA synthesized using SuperScript II reverse transcriptase and oligo(dT) primers.

List of RT- and qRT-PCR primers:

\begin{tabular}{|c|c|}
\hline Primer name & Sequence \\
\hline Adam22-FAM-fw & $\begin{array}{l}\text { CGTCGCCGTCCAGCTCGACCAGGGAAT } \\
\text { AATTGCCGGCACCAT }\end{array}$ \\
\hline Adam22-Rv & GCGAGGTCTCCCATTTTCAC \\
\hline Anks1b-FAM-Fw & $\begin{array}{l}\text { CGTCGCCGTCCAGCTCGACCAGGCTCC } \\
\text { CTAGACGTTCCTCAC }\end{array}$ \\
\hline Anks1b-FAM-Fw & GGATGATGCTGCCAGTACTG \\
\hline Sez6-FAM-Fw & $\begin{array}{l}\text { CGTCGCCGTCCAGCTCGACCAGCCACC } \\
\text { ATCCACTTCTCCTGT }\end{array}$ \\
\hline Sez6-Rev & GCTCCCTAGACGTTCCTCAC \\
\hline Dlg3-FAM-Fw & $\begin{array}{l}\text { CGTCGCCGTCCAGCTCGACCAGTTCCCT } \\
\text { GGGTTAAGTGACGA }\end{array}$ \\
\hline Dlg3-Rev & TCATCGTTGACTCGGTCCTT \\
\hline
\end{tabular}




\begin{tabular}{|c|c|}
\hline $\begin{array}{l}\text { Syngap1-FAM- } \\
\text { Fw }\end{array}$ & $\begin{array}{l}\text { CGTCGCCGTCCAGCTCGACCAGAACATC } \\
\text { CAAAGGCAGCCAAG }\end{array}$ \\
\hline Syngap1-Rev & GCCGGCTCACATAGAAAAGG \\
\hline Prkrir-FAM-Fw & $\begin{array}{l}\text { CGTCGCCGTCCAGCTCGACCAGGGGTT } \\
\text { GAGAATTGTAGGAGAGC }\end{array}$ \\
\hline Prkrir--Rev & CTGCTATGCGGGTTGTTCAA \\
\hline Sorbs2-FAM-Fw & $\begin{array}{l}\text { CGTCGCCGTCCAGCTCGACCAGCGATC } \\
\text { GGAGCCAAGGAGTAT }\end{array}$ \\
\hline Sorbs2-Rev & AGGCTTCTGTCTATGGAGGAC \\
\hline Nrxn1-FAM-Fw & $\begin{array}{l}\text { CGTCGCCGTCCAGCTCGACCAGACACCT } \\
\text { GATGATGGGCGAC }\end{array}$ \\
\hline Nrxn1-Rev & TGAAGCATCAGTCCGTTCCT \\
\hline Ezh2-FAM-Fw & $\begin{array}{l}\text { CGTCGCCGTCCAGCTCGACCAGTGAGA } \\
\text { AGGGACCGGTTTGTT }\end{array}$ \\
\hline Ezh2-Rev & GCATTCAGGGTCTTTAACGGG \\
\hline Triobp-FAM-Fw & $\begin{array}{l}\text { CGTCGCCGTCCAGCTCGACCAGACCCTA } \\
\text { GCCAATGGACACAG }\end{array}$ \\
\hline Triobp-Rev & CTTGAAGTTGAGCAGATCGGG \\
\hline Itch-FAM-Fw & $\begin{array}{l}\text { CGTCGCCGTCCAGCTCGACCAGTGCATT } \\
\text { TCACAGTGGCCTTC }\end{array}$ \\
\hline Itch-Rev & CCCATGGAATCAAGCTGTGG \\
\hline
\end{tabular}

\section{- Bioinformatics}

Downstream computational analysis were performed at the NYULMC Genome Technology Center. All the reads were mapped to the mouse reference genome (mm10) using the STAR aligner (Dobin et al., 2013). Quality control of RNAseq libraries (i.e. the mean read insert sizes and their standard deviations) was calculated using Picard tools (v.1.126) (http://broadinstitute.github.io/picard/). The Read Per Million (RPM) normalized BigWig files were generated using BEDTools (v2.17.0) (Quinlan and Hall, 2010) and bedGraphToBigWig tool (v4). For the SST cIN P8 ECS, approx. 60E6-80E6 reads were aligned per sample; for P8 SST-Nova1, SST-Nova2, SST-dKO, approx. 60E6-70E6 reads were aligned per sample; for P8 SST-cIN wt ECS and SST-Nova-dKO ECS, approx. 60E6-80E6 reads were aligned per sample. The samples processed for downstream analysis were as follows: 9 samples for SST cIN +ECS versus SST cIN ctl at P8 (4/5 samples per condition), 6 samples for SST-Nova1 removal versus 
SST cIN ctl (3 samples per genotype), 6 samples for SST-Nova2 removal versus SST cIN ctl (3 samples

per genotype), 6 samples for SST-dKO removal versus SST cIN ctl (3 samples per genotype) and 7 samples for SST-dKO removal ECS versus SST cIN ctl ECS (4 control samples, 3 cKO samples).

We used rMATS (v3.0.9) (Shen et al., 2014) to quantify the AS event types (i.e.skipped exons (SE), alternative $3^{\prime}$ splice sites (A3SS), alternative 5' splice sites (A5SS), mutually exclusive exons (MXE) and retained introns (RI)). rMATS uses a counts-based model, it detects AS events using splice junction and exon body counts and calculates an exon inclusion level value $\psi$ for each event in each condition. It then determines the differential $|\Delta \psi|$ value across conditions (cut-offs for significance were placed at FDR $<0.05$ and $|\Delta \psi| \geq 0.1)$. To compare the level of similarity among the samples and their replicates, we used two methods: classical multidimensional scaling or principal-component analysis and Euclidean distance-based sample clustering. The downstream statistical analyses and generating plots were performed in Rstudio (Version 1.1.456) (http://www.r- project.org/).

To assess the enrichment for the Nova-binding motif in the differentially regulated exons we utilized rMAPS (Park et al., 2016). We utilized the raw output from rMATS analysis (6 RNAseq experiments of SST cINs +ECS vs SST cINs ctl) with significant splicing events cut off at FDR>50\%. rMAPS performs position weight analysis to assess the enrichment of RNA binding protein binding motifs in the exonic and flanking intronic regions of up-regulated or down-regulated exons and plots the motif density along with a given pValue in comparison to unregulated exons.

We performed GO analysis using the DAVID online Bioinformatics Resources 6.8 at FDR $>0.05$ (unless otherwise specified) (Huang et al., 2008) and tested PPI networks by utilizing DAPPLE at 10,000 permutations (Rossin et al., 2011).

\section{- Validation of SST-cINs AS activity-dependent exons by RT-PCR}

Total RNAs from sorted cINs from wt SST cINs, ECS SST cINs, and ECS SST-dKO were extracted as described above and at least three independent biological replicates were used in each experiment. RT-PCR validation of regulated exons was performed as described before (Han et al., 2014). After 
denaturation, samples were run on $10 \%$ Novex $^{\text {TM }}$ TBE-Urea Gels (ThermoFisher). Gels were directly scanned by ChemiDoc ${ }^{\mathrm{TM}}$ Imaging System (Bio-Rad) and quantified by ImageStudio program (Licor).

\section{QUANTIFICATION AND STATISTICAL ANALYSIS}

In all figures: ${ }^{*}, p$-value $<0.05 ;{ }^{* *}, p$-value $<0.01 ;{ }^{* * *}, p$-value $<0.001 ;{ }^{* * * *}, p$-value $<0.0001$. Statistical analyses for motif enrichment was performed by rMAPS and differential alternative splicing changes were performed using rMATS. Percentages were compared with repeated t-tests in GraphPad Prism or Rstudio, and means \pm (standard deviation, SD) are represented. As well as, some statistical analyses and generating plots were performed in R environment (v3.1.1) (http://www.r- project.org/).

All values presented in the manuscript are average \pm standard error of the mean (SEM). The statistical values for the intrinsic physiology are obtained using one-way ANOVA with Bonferroni correction for multiple comparisons between the different genotypes: Controls, Nova1-cKO, Nova2-cKO and NovadKO $\left({ }^{*} p \leq 0.05,{ }^{* *} p \leq 0.01,{ }^{* *} p \leq 0.005\right)$. For the Channelrhodopsin output, student's t-test was used to compare Control vs Nova1-cKO, and Control vs Nova2-cKO $\left({ }^{*} p \leq 0.05,{ }^{* *} p \leq 0.01,{ }^{* * *} p \leq 0.005\right)$.

DATA AND SOFTWARE AVAILABILITY The accession number for the RNA sequencing data reported in this paper is NCBI GEO: [TBD].

\section{Figure legends}

\section{Figure 1. Neuronal activity affects the synaptic development of SST cINs}

A) Schematic of genetic alleles (left) and experimental approach (middle), SSTCre;Syp-eGFP pups were injected with a conditional virus; either AAV2/1-Flex-Kir2.1-P2A-mCherry, AAV2/1-FlexNaChBac-P2A-mCherry, or Control AAV2/1-Flex-mCherry within the S1 cortex at postnatal day 0 (P0). Schematic of the efferent connectivity of SST+ Martinotti cells (right).

B) Immunostaining (IHC) of SSTCre;Syp-eGFP in layer 1 (L1) of S1 cortex at P21 showing Syp-eGFP (green, anti-GFP) and axons (red, anti-RFP) from control, Kir2.1, or NaChBac injected SST+ Marintonotti clNs (scale bar 50um). 
C) Quantification of SST cIN axonal density within L1 of somatosensory cortex of control, KIR2.1, and

NaChBac injected SSTcre animals $\left(p \mathrm{pal}^{*}=0.011,{ }^{* * *}=0.0008\right)$

D) Visualization of Syp-eGFP (green, anti-GFP) and Gephyrin+ puncta (blue, anti-Gephyrin) in L1 SST+ clNs (scale bar 20um). Inset shows a higher magnification image of the puncta overap (Red, mCherry axons).

E) Quantification of synaptic puncta (RFP+/GFP+/Gephyrin+ overlap) of control, Kir2.1, and NaChBac expressing SST+ cINs within L1 $\left(\mathrm{pVal}^{* *}=0.008,{ }^{* * *}=0.0001\right)$

Figure 2. Neuronal activity influences alternative splicing and Nova expression within SST cINs A) Schematic of experimental approach: Postnatal day 8 (P8) SSTCre;RCE ${ }^{\text {eGFP }}$ or SSTCre;Ai9 pups were to electroconvulsive shock (ECS) (left). Following 2-3 hours the S1 cortex was isolated and SST+ cINs were FACS purified (middle). SST+ cINs were then prepared for RNAseq to assess changes in alternative splicing. Splicing changes are divided into the major alternative structural motifs: single exon, SE, retained intron, RI, mutually exclusive exons, MXE, alternative 5' splice site, A5, alternative 3' splice site, A3 (right).

B) Histogram of the magnitude of activity-dependent splicing changes within SST+ cINs subjected to ECS compared to sham SST+ cINs (FDR <0.5, fold $<0.1>$ ), depicting 139 differential spliced SE (82 SE included, 57 SE excluded), 66 differential spliced RI (53 RI included, 13 RI excluded), 55 differential spliced MXE (26 included, 29 excluded), 13 differential spliced A5 (12 included, 1 excluded), 39 differential spliced A3 (16 included, 23 excluded).

C) Sashimi plot illustrating Nxrn1 exon 10 exclusion in activity-induced SST cINs in green (bottom) compared to sham SST cINs in grey (top). Reads per kilobase of transcripts (RPKM) gives the count of the number of transcripts for a specfic isoform.

D) Histogram of the average motif enrichment score of known activity-regulated splicing factors KHDRBS1 (Sam68), KHDRBS2 (SLM2), Rbfox1 and Nova1/2 (right). Green dots represents -log10 adjusted $\mathrm{p}$ value (right $\mathrm{Y}$-axis) for motif enrichment scores, only significant enrichment shown. 
E) Bubble dot plot of gene ontology (GO) most significant terms for the genes subjected to activitydependent alternative splicing within SST+ cINs (false discovery rate $(F D R)<0.05$ ), $x$-axis is the enrichment of the activity-dependent AS genes in the GO catagory (\# of genes in GO category from SST transcriptome/ \# of genes activity-dependent AS in category). Color of dot indicates magnitude of significance $(-\log 10$ transform FDR, none shown above FDR <0.05) and size corresponds to number of genes in catagory. F) Protein-protein interaction (PPI) network formed from 312 activity-depdendent spliced genes in SST cINs with Disease Association Protein-Protein Link Evaluator (DAPPLE) (Rossin et al., 2011) and performed over 10,000 permutations (pVal<0.00009). Green shading- post-synaptic gene network, pink shading- pre-synaptic gene network.

Figure 3. Neuronal activity during cortical development influences the expression and localization of Nova proteins in SST cINs.

A) Relative gene expression of Nova1 (orange) and Nova2 (pink), normalized to house-keeping gene Peptidyl prolyl isomerase A (PPIA) using qPCR from Lhx6-eGFP sorted cINs at Postnatal age (P) P2, P8 and P15.

B) Fold change of the relative expression of Nova1 and Nova2 between cINs and excitatory neurons (cExt) showing an enrichment of Nova expression in cINs at early developmental ages.

C) Model of experimental approach: Acute activity induction via electrical stimulation (ECS) followed by qPCR 1.5 hrs post stimulation (HRPS), Western and IHC 2.5HRPS at P8.

D) Relative expression of Nova1 and Nova2 genes (using qPCR) of ECS induced SST cINs relative to controls $\left({ }^{* *} \mathrm{pVal}=0.002\right.$, Nova1; ${ }^{* *} \mathrm{pVal}=0.005$, Nova2).

E) Upper panel, western blot showing Nova1 and Nova2 protein expression in control (lanes 2 and 3) versus ECS induced SST cINs (lanes 4 and 5). Lower panel, same western blot showing expression of b-actin across lanes.

F) Quantification of the western blot data. Nova1 and Nova2 protein expression relative to b-actin in control versus ECS induced SST cINs ( ${ }^{*} \mathrm{pVal}=0.038$, Nova1; ${ }^{*} \mathrm{pVal}=0.022$, Nova2). 
G) Representative scoring criteria for Nova1/2 localization within SST cINs: IHC of Nova1/2 (blue, antiNova1/2) in selective SST+ cINS exemplifying the Nova1/2 expression in: cytoplasm only (top), in both cytoplasm and nucleus (middle), and nucleus only (bottom).

H) Representative images of Nova1/2 expression in SST cINs under normal versus ECS

I) Quantification of the ratio of nuclear to cytoplasmic localization of Nova1/2 in SST+ clNs of control animals (grey) and ECS animals (black) ${ }^{* *} \mathrm{pVal}=0.001$.

J) Schematic of the constitutive activity manipulation via injection of DIO:AAVs (AAV2/1-Flex-mCherry, AAV2/1-Flex-NaChBac-P2A-mCherry, and AAV2/1Flex-Kir2.1-P2A-mCherry) at P0 followed by IHC at P21.

K) Left, Quantification of the ratio of nuclear to cytoplasmic localization of Nova1/2 in SST+ cINs of control AAV2/1-Flex-mCherry (grey) versus AAV2/1-Flex-NaChBac-P2A-mCherry. Right, Quantification of the ratio of nuclear to cytoplasmic localization of Nova1/2 in SST+ cINs of control (grey) AAV-2/1-Flex-mCherry (grey) versus AAV2/1-Flex-Kir2.1-P2A-mCherry (blue) injected animals. ${ }^{* * *}$ pVal=0.0004, NachBac; ${ }^{* *} p$ Val=0.0001, KIR2.1

L) Quantification of the number of Nova1/2-expressing SST+ cINs of control AAV2/1-Flex-mCherry (grey) and AAV2/1-Flex-Kir2.1- P2A-mCherry (blue) injected animals. ${ }^{* *}$ pVal= 0.0001

M) Representative images of Nova1/2 expression, Top: control SST cIN (injected with mCherry), Bottom: KIR2.1+ SSt cIN at P21.

N) Quantification of Nova1/2 protein pixel intensity (normalized to area) from ctl SST cINs (grey) and KIR2.1+ SST cINs (blue).

Figure 4. SST-Nova1 and SST-Nova2 mutants have impaired afferent and efferent connectivity. A) SST+ cINs efferent structure: IHC of anti-RFP (red), anti-VGAT (green), and anti-Gephyrin (blue) to label the SST+ cIN axonal synaptic puncta (RFP+/VGAT+/Gephyrin+ puncta, white) in L1 S1 cortex of SST-ctl, SST-Nova1, and SST-Nova2 mutant animals. 
B) Quantification of the density of SST+ cIN efferent synaptic puncta (RFP+/VGAT+/Gephyrin+) in L1

S1 cortex of SST-ctl, SST-Nova1 and SST-Nova2 mutant animals. ${ }^{* *}$ pVal=0.003, SST-Nova1; ${ }^{* * *} \mathrm{pVal}<0.0001$, SST-Nova2

C) Schematic of channelrhodopsin (ChR2) experimental approach: SST-Cre control, SST-Nova1 or SST-Nova2 mutant mice were crossed with the Ai32 reporter line that expresses ChR2 in a Cre dependent manner. Blue light was delivered through the objective to record inhibtory response (IPSC) in neighboring excitatory neuron (grey).

D) Top: Example trace of inhibitory output from control SST+ cIN within S1 of SST-ctl (grey trace), and SST-Nova1 mutant (orange trace). Bottom: control SST+ cIN within S1 (grey trace), SST-Nova2 (pink trace) animals. Right: Quantification of the peak IPSC amplitudes recorded in excitatory neurons following SST stimulation. **pVal=0.0037, SST-Nova1; **pVal=0.0021, SST-Nova2.

E) SST+ afferents: IHC of representative SST+ cIN dendrite of anti-RFP (red), anti-VGLUT1 (green), and anti-Homer1c (blue) to label excitatory synaptic puncta overlapping with SST+ cINs dendrites (RFP+/VGLUT1+/Homer1c+ puncta, white) in S1 cortex of SST-ctl, SST-Nova1 and SST-Nova2 mutant animals.

F) Quantification of the density of excitatory afferent synapses onto SST+ cINs within L2/3 and L5/6 of S1 cortex of SST-ctl, SST-Nova1 and SST-Nova2 mutant animals. ${ }^{*}$ pVal=0.028, SST-Nova1; ${ }^{*} \mathrm{pVal}=0.012$, SST-Nova2.

G) Schematic of experimental approach recording mini-excitatory postsynaptic potentials (mEPSCs) in SST+ cINs in the S1 cortex (red).

H) Quantification of mEPSCs frequencies from SST+ cIN CtI,SST-Nova1 and SST-Nova2 mutant animals.

Figure 5. Nova RNA binding proteins control activity dependent AS in SST cINS during development

A) Schematic of experimental approach: Control and SST-dKO P8 animals were subjected to ECS then the S1 cortex was isolated to FACS purify SST+ cINs followed by RNAseq and splicing analysis. 
B) Magnitude of activity-dependent splicing changes within SST-dKO subjected to ECS compared to

Ctr SST-wt cINs subjected to ECS (FDR <0.5, fold <0.1>), depicting 166 differential spliced SE (106 SE included, 60 SE excluded), 72 differential spliced RI (51 RI included, 21 RI excluded), 70 differential spliced MXE (37 included, 33 excluded), 9 differential spliced A5 (7 included, 2 excluded), 29 differential spliced A3 (9 included, 20 excluded).

C) Example RT-PCR validation of alternative splicing (AS) events of activity- and Nova1/2- dependent alternative exon usage within the gene Syngap1 (left), Gel image of RT-PCR product from the amplification of exon13 to exon 15 within SST-ctl cINs (CtI) (left), ECS-treated Ctl (middle), and ECStreated SST-dKO (right).

D) Quantification of RT-PCR AS events of Syngap1. ${ }^{* *}$ pVal=0.0001 Ctl vs Ctl+ECS; ${ }^{* *}$ pVal=0.004 Ctl+ECS vs SST-dKO+ECS.

E) Bubble dot plot of the most significant GO terms for the genes undergoing Nova1/2 activitydependent AS within SST+ cINs (all shown GO terms FDR<0.05) (Same graph parameters as Figure2E).

F) Schematic of a SST+ cIN pre-synaptic inhibitory axonal puncta (top right) and a SST+ cIN excitatory post-synaptic density (middle left) overlaid on top of the significant DAPPLE generated PPI diect network from the 346 genes undergoing Nova1/2-dependent activity induced AS $\left({ }^{* * *} p\right.$ Val=0.00009, 10000 permutations).

Figure 6. Nova2 overexpression within SST cINs augments axonal growth and synaptic formation in an activity dependent manner.

A) Experimental model: Schematic of genetic alleles (left) crossed to generate mice for injection of AAV2/1-Flex-Syp-eGFP with AAV2/1-TRE-Nova2-mCherry, or AAV2/1-Flex-Syp-eGFP, AAV2/1-TRENova2-mCherry and AAV2/1-Flex-KIR2.1 at P0 followed by IHC at P21.

B) IHC of SST+ cINs axons labeled by Syp-eGFP (Anti-GFP) in control, C) Nova2-OE, and D) Nova2OE+KIR2.1 injected S1 cortex.

E) Quantification of axonal density of control (grey), Nova2-OE (pink), and Nova2-OE+KIR2.1 (blue) 
injected SST + cINs pVal ${ }^{*} \leq 0.05,{ }^{* *} \leq 0.01,{ }^{* * *} \leq 0.005$

F) Representative images of SST+ cIN axonal efferents within L1 of S1 cortex labeled by Syp-eGFP (anti-GFP, green) and gephryn (anti-gephryn, blue) in Control, G) Nova2OE, and H) Nova2OE+KIR2.1.

I) Quantification of L1 SST+ cIN efferent synaptic puncta via the juxapositon of Syp-eGFP and Gephryn in control (grey), Nova2OE (pink), and Nova2OE +KIR2.1 (blue).

J) Representative images of IHC against mCherry (anti-RFP, red), and Nova1/2 (anti-Nova1/2, blue) in two SST-Nova2OE cells and K) in two SST-Nova2OE+KIR2.1 cells. Top is merge (red/blue) and botton is Nova1/2 (blue) alone.

L) Quantification of the Ratio of Nova1/2 localization within the nucleus to cytoplasm from Nova2OE SSt cINs (pink) and Nova2OE+KIR2.1 (blue). pVal= ${ }^{*} \leq 0.05,{ }^{* *} \leq 0.01,{ }^{* * *} \leq 0.005$.

M) Model of experimental findings: center is a cartoon wildtype SST cIN depicting normal expression of Nova1/2 with the soma (red) whereas, on the left, the conditional loss of Nova1, Nova2, or the expression of KIR2.1 alone or dual overexpression of Nova2 and KIR2.1 results in the reduction in Nova expression and restricts Nova localization to the cytoplasm (In the case of cKO animals the protein is lost completely). This effect is accompanied by a reduction in the connectivity of SST cINs.

To the contrary, Expression of NaChBac and/or overexpression of Nova2 alone results in expression of Nova throughout the cell and nucleus and is accompanied by an increase in the axonal and synaptic density of SST cINs.

\section{References}

Adler, A., Zhao, R., Shin, M.E., Yasuda, R., Gan, W.-B., 2019. Somatostatin-Expressing Interneurons Enable and Maintain Learning-Dependent Sequential Activation of Pyramidal Neurons. Neuron 123. doi:10.1016/j.neuron.2019.01.036

Allene, C., Cattani, A., Ackman, J.B., Bonifazi, P., Aniksztejn, L., Ben-Ari, Y., Cossart, R., 2008. Sequential Generation of Two Distinct Synapse-Driven Network Patterns in Developing Neocortex. Journal of Neuroscience 28, 12851-12863. doi:10.1523/JNEUROSCI.3733-08.2008

Allene, C., Cossart, R., 2010. Early NMDA receptor-driven waves of activity in the developing neocortex: physiological or pathological network oscillations? The Journal of Physiology 588, 8391. doi:10.1113/jphysiol.2009.178798

Basaldella, E., Takeoka, A., Sigrist, M., Arber, S., 2015. Multisensory Signaling Shapes VestibuloMotor Circuit Specificity. Cell 163, 301-312. doi:10.1016/j.cell.2015.09.023 
bioRxiv preprint doi: https://doi.org/10.1101/845230; this version posted November 16,2019 . The copyright holder for this preprint (which was not certified by peer review) is the author/funder, who has granted bioRxiv a license to display the preprint in perpetuity. It is made available under aCC-BY-NC-ND 4.0 International license.

Bortone, D., Polleux, F., 2009. KCC2 Expression Promotes the Termination of Cortical Interneuron Migration in a Voltage-Sensitive Calcium-Dependent Manner. Neuron 62, 53-71. doi:10.1016/j.neuron.2009.01.034

Cossart, R., 2011. The maturation of cortical interneuron diversity: how multiple developmental journeys shape the emergence of proper network function. Curr. Opin. Neurobiol. 21, 160-168. doi:10.1016/j.conb.2010.10.003

De Marco García, N.V., Karayannis, T., Fishell, G., 2011. Neuronal activity is required for the development of specific cortical interneuron subtypes. Nature 472, 351-355.

doi:10.1038/nature09865

Denaxa, M., Neves, G., Rabinowitz, A., Kemlo, S., Liodis, P., Burrone, J., Pachnis, V., 2018. Modulation of Apoptosis Controls Inhibitory Interneuron Number in the Cortex. Cell Reports 22, 1710-1721. doi:10.1016/j.celrep.2018.01.064

Dredge, B.K., Darnell, R.B., 2003. Nova regulates GABA(A) receptor gamma2 alternative splicing via a distal downstream UCAU-rich intronic splicing enhancer. Molecular and Cellular Biology 23, 4687-4700. doi:10.1128/mcb.23.13.4687-4700.2003

Eom, T., Zhang, C., Wang, H., Lay, K., Fak, J., Noebels, J.L., Darnell, R.B., 2013. NOVA-dependent regulation of cryptic NMD exons controls synaptic protein levels after seizure. Elife 2, 5752-29. doi:10.7554/eLife.00178

Favuzzi, E., Deogracias, R., Marques-Smith, A., Maeso, P., Jezequel, J., Exposito-Alonso, D., Balia, M., Kroon, T., Hinojosa, A.J., F Maraver, E., Rico, B., 2019. Distinct molecular programs regulate synapse specificity in cortical inhibitory circuits. Science 363, 413-417. doi:10.1126/science.aau8977

Fishell, G, Rudy, B., 2011. Mechanisms of Inhibition within the Telencephalon: "Where the Wild Things Are." Annu. Rev. Neurosci. 34, 535-567. doi:10.1146/annurev-neuro-061010-113717

Furlanis, E., Scheiffele, P., 2018. Regulation of Neuronal Differentiation, Function, and Plasticity by Alternative Splicing. Annu. Rev. Cell Dev. Biol. 34, 451-469. doi:10.1146/annurev-cellbio-100617062826

Furlanis, E., Traunmüller, L., Fucile, G., Scheiffele, P., 2019. Landscape of ribosome-engaged transcript isoforms reveals extensive neuronal-cell-class- specific alternative splicing programs. Nature Neuroscience 1-15. doi:10.1038/s41593-019-0465-5

Garaschuk, O., Linn, J., Eilers, J., Konnerth, A., 2000. Large-scale oscillatory calcium waves in the immature cortex. Nature Neuroscience 3, 452-459. doi:10.1038/74823

Gong, S., Zheng, C., Doughty, M.L., Losos, K., Didkovsky, N., Schambra, U.B., Nowak, N.J., Joyner, A., Leblanc, G., Hatten, M.E., Heintz, N., 2003. A gene expression atlas of the central nervous system based on bacterial artificial chromosomes. Nature Publishing Group 425, 917-925. doi:10.1038/nature02033

Guo, J.U., Ma, D.K., Mo, H., Ball, M.P., Jang, M.-H., Bonaguidi, M.A., Balazer, J.A., Eaves, H.L., Xie, B., Ford, E., Zhang, K., Ming, G.-L., Gao, Y., Song, H., 2011. Neuronal activity modifies the DNA methylation landscape in the adult brain. Nature Neuroscience 14, 1345-1351. doi:10.1038/nn.2900

lijima, T., Wu, K., Witte, H., Hanno-lijima, Y., Glatter, T., Richard, S., Scheiffele, P., 2011a. SAM68 regulates neuronal activity-dependent alternative splicing of neurexin-1. Cell 147, 1601-1614. doi:10.1016/j.cell.2011.11.028

lijima, T., Wu, K., Witte, H., Hanno-lijima, Y., Glatter, T., Richard, S., Scheiffele, P., 2011b. SAM68 regulates neuronal activity-dependent alternative splicing of neurexin-1. Cell 147, 1601-1614. doi:10.1016/j.cell.2011.11.028

Ippolito, D.M., Eroglu, C., 2010. Quantifying synapses: an immunocytochemistry-based assay to quantify synapse number. J Vis Exp. doi:10.3791/2270

Kapfer, C., Glickfeld, L.L., Atallah, B.V., Scanziani, M., 2007. Supralinear increase of recurrent inhibition during sparse activity in the somatosensory cortex. Nature Neuroscience 10, 743-753. doi:10.1038/nn1909 
bioRxiv preprint doi: https://doi.org/10.1101/845230; this version posted November 16,2019 . The copyright holder for this preprint (which was not certified by peer review) is the author/funder, who has granted bioRxiv a license to display the preprint in perpetuity. It is made available under aCC-BY-NC-ND 4.0 International license.

Karayannis, T., De Marco García, N.V., Fishell, G.J., 2012. Functional adaptation of cortical interneurons to attenuated activity is subtype-specific. Front Neural Circuits 6, 66. doi:10.3389/fncir.2012.00066

Kepecs, A., Fishell, G, 2014. Interneuron cell types are fit to function. Nature 505, 318-326. doi:10.1038/nature12983

Le Magueresse, C., Monyer, H., 2013. GABAergic Interneurons Shape the Functional Maturation of the Cortex. Neuron 77, 388-405. doi:10.1016/j.neuron.2013.01.011

Lee, J.-A., Xing, Y., Nguyen, D., Xie, J., Lee, C.J., Black, D.L., 2007. Depolarization and CaM Kinase IV Modulate NMDA Receptor Splicing through Two Essential RNA Elements. PLoS Biol 5, e4014. doi:10.1371/journal.pbio.0050040

Lee, J.A., Tang, Z.Z., Black, D.L., 2009. An inducible change in Fox-1/A2BP1 splicing modulates the alternative splicing of downstream neuronal target exons. Genes \& Development 23, 2284-2293. doi:10.1101/gad.1837009

Li, L., Tasic, B., Micheva, K.D., Ivanov, V.M., Spletter, M.L., Smith, S.J., Luo, L., 2010. Visualizing the Distribution of Synapses from Individual Neurons in the Mouse Brain. PLoS ONE 5, e11503-13. doi:10.1371/journal.pone.0011503

Licatalosi, D.D., Mele, A., Fak, J.J., Ule, J., Kayikci, M., Chi, S.W., Clark, T.A., Schweitzer, A.C., Blume, J.E., Wang, X., Darnell, J.C., Darnell, R.B., 2008. HITS-CLIP yields genome-wide insights into brain alternative RNA processing. Nature 456, 464-469. doi:10.1038/nature07488

Lim, L., Mi, D., Llorca, A., Marín, O., 2018. Development and Functional Diversification of Cortical Interneurons. Neuron 100, 294-313. doi:10.1016/j.neuron.2018.10.009

Lin, C.-W., Sim, S., Ainsworth, A., Okada, M., Kelsch, W., Lois, C., 2010. Genetically increased cellintrinsic excitability enhances neuronal integration into adult brain circuits. Neuron 65, 32-39. doi:10.1016/j.neuron.2009.12.001

Liu, Z., Wang, L., Welch, J.D., Ma, H., Zhou, Y., Vaseghi, H.R., Yu, S., Wall, J.B., Alimohamadi, S., Zheng, M., Yin, C., Shen, W., Prins, J.F., Liu, J., Qian, L., 2017. Single-cell transcriptomics reconstructs fate conversion from fibroblast to cardiomyocyte. Nature Publishing Group 551, 100104. doi:10.1038/nature24454

Ma, D.K., Jang, M.H., Guo, J.U., Kitabatake, Y., Chang, M.L., Pow-anpongkul, N., Flavell, R.A., Lu, B., Ming, G.L., Song, H., 2009. Neuronal Activity-Induced Gadd45b Promotes Epigenetic DNA Demethylation and Adult Neurogenesis. Science 323, 1074-1077. doi:10.1126/science.1166859

Marín, O., 2016. Developmental timing and critical windows for the treatment of psychiatric disorders. Nature Publishing Group 22, 1229-1238. doi:10.1038/nm.4225

Mauger, O., Lemoine, F., Scheiffele, P., 2016. Targeted Intron Retention and Excision for Rapid Gene Regulation in Response to Neuronal Activity. Neuron 92, 1266-1278. doi:10.1016/j.neuron.2016.11.032

Minlebaev, M., Colonnese, M., Tsintsadze, T., Sirota, A., Khazipov, R., 2011. Early y oscillations synchronize developing thalamus and cortex. Science 334, 226-229. doi:10.1126/science.1210574

Muñoz, W., Tremblay, R., Levenstein, D., Rudy, B., 2017. Layer-specific modulation of neocortical dendritic inhibition during active wakefulness. Science 355, 954-959. doi:10.1126/science.aag2599

Nigro, M.J., Hashikawa-Yamasaki, Y., Rudy, B., 2018. Diversity and Connectivity of Layer 5 Somatostatin-Expressing Interneurons in the Mouse Barrel Cortex. Journal of Neuroscience 38, 1622-1633. doi:10.1523/JNEUROSCI.2415-17.2017

Park, J.W., Jung, S., Rouchka, E.C., Tseng, Y.-T., Xing, Y., 2016. rMAPS: RNA map analysis and plotting server for alternative exon regulation. Nucleic Acids Res 44, W333-W338. doi:10.1093/nar/gkw410

Priya, R., Paredes, M.F., Karayannis, T., Yusuf, N., Liu, X., Jaglin, X., Graef, I., Alvarez-Buylla, A., Fishell, G., 2018. Activity Regulates Cell Death within Cortical Interneurons through a CalcineurinDependent Mechanism. Cell Reports 22, 1695-1709. doi:10.1016/j.celrep.2018.01.007 
bioRxiv preprint doi: https://doi.org/10.1101/845230; this version posted November 16,2019 . The copyright holder for this preprint (which was not certified by peer review) is the author/funder, who has granted bioRxiv a license to display the preprint in perpetuity. It is made available under aCC-BY-NC-ND 4.0 International license.

Quesnel-Vallières, M., Dargaei, Z., Irimia, M., Gonatopoulos-Pournatzis, T., Ip, J.Y., Wu, M., SterneWeiler, T., Nakagawa, S., Woodin, M.A., Blencowe, B.J., Cordes, S.P., 2016. Misregulation of an Activity-Dependent Splicing Network as a Common Mechanism Underlying Autism Spectrum Disorders. Molecular Cell 64, 1023-1034. doi:10.1016/j.molcel.2016.11.033

Racca, C., Gardiol, A., Eom, T., Ule, J., Triller, A., Darnell, R.B., 2010. The Neuronal Splicing Factor Nova Co-Localizes with Target RNAs in the Dendrite. Front Neural Circuits 4, 5. doi:10.3389/neuro.04.005.2010

Rossin, E.J., Lage, K., Raychaudhuri, S., Xavier, R.J., Tatar, D., Benita, Y., International Inflammatory Bowel Disease Genetics Constortium, Cotsapas, C., Daly, M.J., 2011. Proteins Encoded in Genomic Regions Associated with Immune-Mediated Disease Physically Interact and Suggest Underlying Biology. PLoS Genet 7, e1001273-13. doi:10.1371/journal.pgen.1001273

Saito, Y., Miranda-Rottmann, S., Ruggiu, M., Park, C.Y., Fak, J.J., Zhong, R., Duncan, J.S., Fabella, B.A., Junge, H.J., Chen, Z., Araya, R., Fritzsch, B., Hudspeth, A.J., Darnell, R.B., 2016. NOVA2mediated RNA regulation is required for axonal pathfinding during development. Elife 5, 487. doi:10.7554/eLife.14371

Saito, Y., Yuan, Y., Zucker-Scharff, I., Fak, J.J., Jereb, S., Tajima, Y., Licatalosi, D.D., Darnell, R.B., 2019. Differential NOVA2-Mediated Splicing in Excitatory and Inhibitory Neurons Regulates Cortical Development and Cerebellar Function. Neuron 1-20. doi:10.1016/j.neuron.2018.12.019 Silberberg, G., Markram, H., 2007. Disynaptic Inhibition between Neocortical Pyramidal Cells Mediated by Martinotti Cells. Neuron 53, 735-746. doi:10.1016/j.neuron.2007.02.012

Sousa, V.H., Miyoshi, G., Hjerling Leffler, J., Karayannis, T., Fishell, G., 2009. Characterization of Nkx6-2-Derived Neocortical Interneuron Lineages. Cerebral Cortex 19, i1-i10. doi:10.1093/cercor/bhp038

Taniguchi, H., He, M., Wu, P., Kim, S., Paik, R., Sugino, K., Kvitsani, D., Fu, Y., Lu, J., Lin, Y., Miyoshi, G., Shima, Y., Fishell, G., Nelson, S.B., Huang, Z.J., 2011. A Resource of Cre Driver Lines for Genetic Targeting of GABAergic Neurons in Cerebral Cortex. Neuron 71, 995-1013. doi:10.1016/j.neuron.2011.07.026

Tremblay, R., Lee, S., Rudy, B., 2016. GABAergic Interneurons in the Neocortex: From Cellular Properties to Circuits. Neuron 91, 260-292. doi:10.1016/j.neuron.2016.06.033

Ule, J., Jensen, K., Mele, A., Darnell, R.B., 2005. CLIP: A method for identifying protein-RNA interaction sites in living cells. Methods 37, 376-386. doi:10.1016/j.ymeth.2005.07.018

Ule, J., Stefani, G., Mele, A., Ruggiu, M., Wang, X., Taneri, B., Gaasterland, T., Blencowe, B.J., Darnell, R.B., 2006. An RNA map predicting Nova-dependent splicing regulation. Nature 444, 580-586. doi:10.1038/nature05304

Vuong, C.K., Black, D.L., Zheng, S., 2016. The neurogenetics of alternative splicing. Nat Rev Neurosci 17, 265-281. doi:10.1038/nrn.2016.27

Vuong, C.K., Wei, W., Lee, J.-A., Lin, C.-H., Damianov, A., la Torre Ubieta, de, L., Halabi, R., Otis, K.O., Martin, K.C., O’Dell, T.J., Black, D.L., 2018. Rbfox1 Regulates Synaptic Transmission through the Inhibitory Neuron-Specific vSNARE Vamp1. Neuron 98, 127-141.e7. doi:10.1016/j.neuron.2018.03.008

Wamsley, B., Fishell, G, 2017. Genetic and activity-dependent mechanisms underlying interneuron diversity. Nature Publishing Group 18, 299-309. doi:10.1038/nrn.2017.30

Wamsley, B., Jaglin, X.H., Favuzzi, E., Quattrocolo, G., Nigro, M.J., Yusuf, N., Khodadadi-Jamayran, A., Rudy, B., Fishell, G., 2018. Rbfox1 Mediates Cell-type-Specific Splicing in Cortical Interneurons. Neuron 100, 846-859.e7. doi:10.1016/j.neuron.2018.09.026

Xie, J., Black, D.L., 2001. A CaMK IV responsive RNA element mediates depolarization-induced alternative splicing of ion channels. Nature 410, 936-939. doi:10.1038/35073593

Yang, J.-W., An, S., Sun, J.-J., Reyes-Puerta, V., Kindler, J., Berger, T., Kilb, W., Luhmann, H.J., 2012. Thalamic Network Oscillations Synchronize Ontogenetic Columns in the Newborn Rat Barrel Cortex. Cerebral Cortex 23, 1299-1316. doi:10.1093/cercor/bhs103 
Yang, J.W., Hanganu-Opatz, I.L., Sun, J.J., Luhmann, H.J., 2009. Three Patterns of Oscillatory Activity Differentially Synchronize Developing Neocortical Networks In Vivo. Journal of Neuroscience 29, 9011-9025. doi:10.1523/JNEUROSCI.5646-08.2009

Yang, Y., Park, J.W., Bebee, T.W., Warzecha, C.C., Guo, Y., Shang, X., Xing, Y., Carstens, R.P., 2016. Determination of a Comprehensive Alternative Splicing Regulatory Network and Combinatorial Regulation by Key Factors during the Epithelial-to-Mesenchymal Transition. Molecular and Cellular Biology 36, 1704-1719. doi:10.1128/MCB.00019-16

Yano, M., Hayakawa-Yano, Y., Mele, A., Darnell, R.B., 2010. Nova2 Regulates Neuronal Migration through an RNA Switch in Disabled-1 Signaling. Neuron 66, 848-858. doi:10.1016/j.neuron.2010.05.007

Yu, C.R., Power, J., Barnea, G., O'Donnell, S., Brown, H.E.V., Osborne, J., Axel, R., Gogos, J.A., 2004. Spontaneous neural activity is required for the establishment and maintenance of the olfactory sensory map. Neuron 42, 553-566. doi:10.1016/s0896-6273(04)00224-7

Yuan, Y., Xie, S., Darnell, J.C., Darnell, A.J., Saito, Y., Phatnani, H., Murphy, E.A., Zhang, C., Maniatis, T., Darnell, R.B., 2018a. Cell type-specific CLIP reveals that NOVA regulates cytoskeleton interactions in motoneurons 1-19. doi:10.1186/s13059-018-1493-2

Yuan, Y., Xie, S., Darnell, J.C., Darnell, A.J., Saito, Y., Phatnani, H., Murphy, E.A., Zhang, C., Maniatis, T., Darnell, R.B., 2018b. Cell type-specific CLIP reveals that NOVA regulates cytoskeleton interactions in motoneurons 1-19. doi:10.1186/s13059-018-1493-2 
bioRxiv preprint doi: https://doi.org/10.1101/845230; this version posted November 16,2019 . The copyright holder for this preprint (which was not certified by peer review) is the author/funder, who has granted bioRxiv a license to display the preprint in perpetuity. It is made available

A Genetic Strategy:

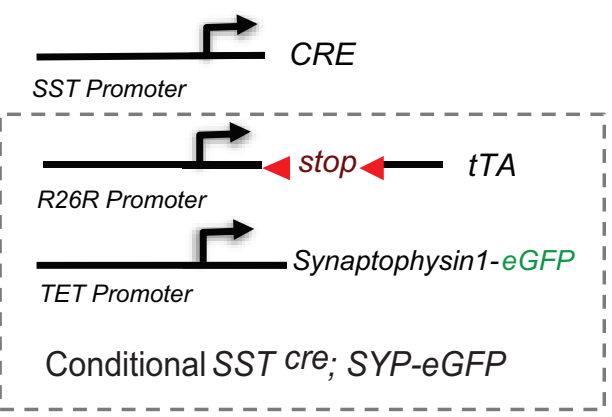
AAV-Fex-mCherry

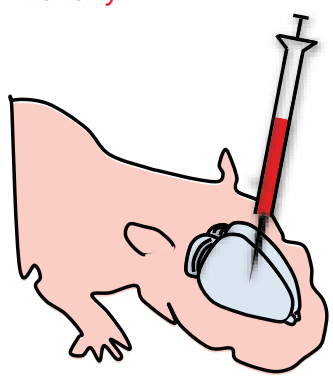

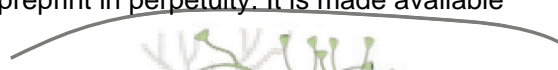

B

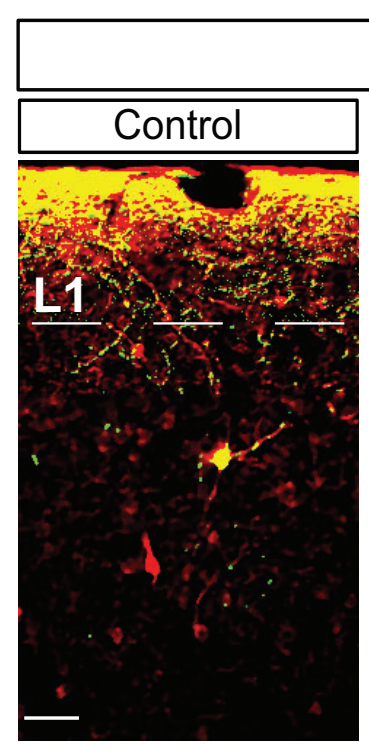

SYPeGFP AAV
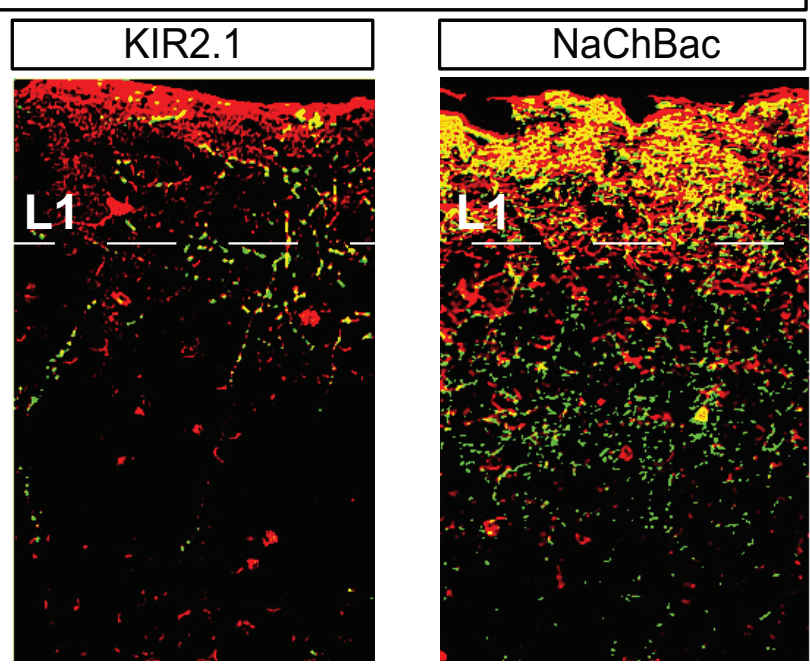

D

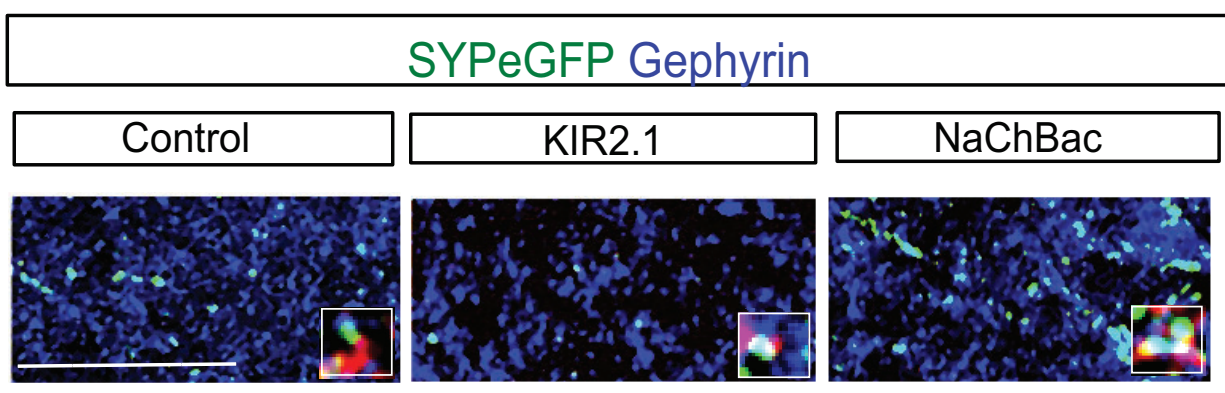

C

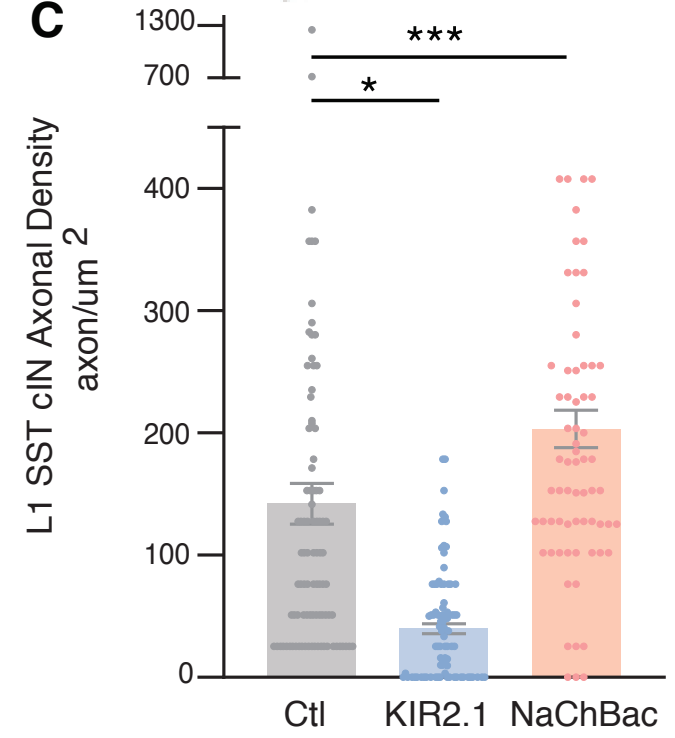

E

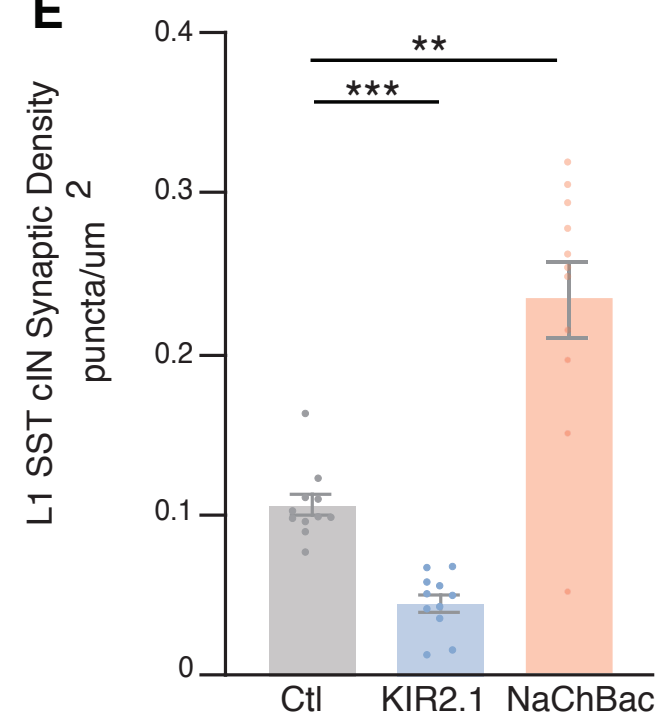


bioRxiv preprint doi: https://doi.org/10.1101/845230; this version posted November 16,2019 . The copyright holder for this preprint (which was not certified by peer review) is the author/funder, who has granted bioRxiv a license to display the preprint in perpetuity. It is made available

A under aCC-BY-NC-ND 4.0 International license.

SSTCre;Aig
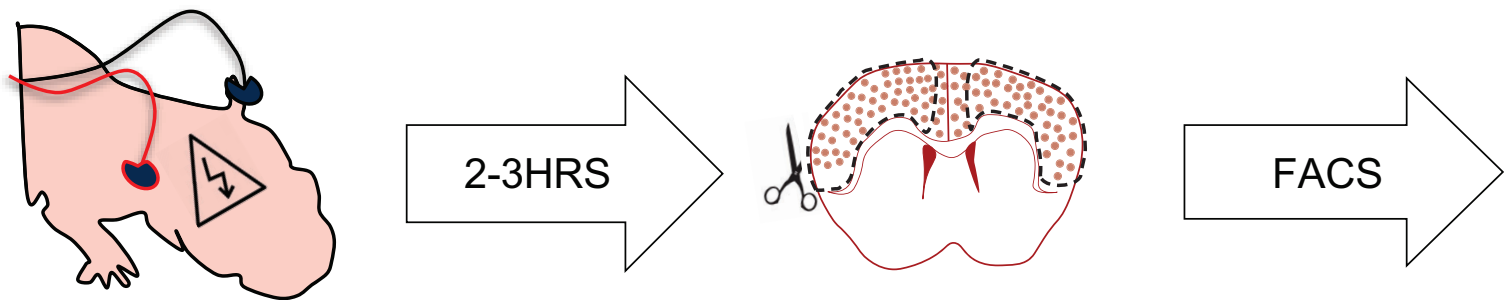

SE

RI

MXE

A5

A3

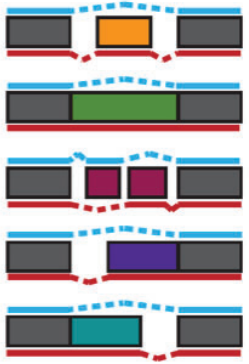

B

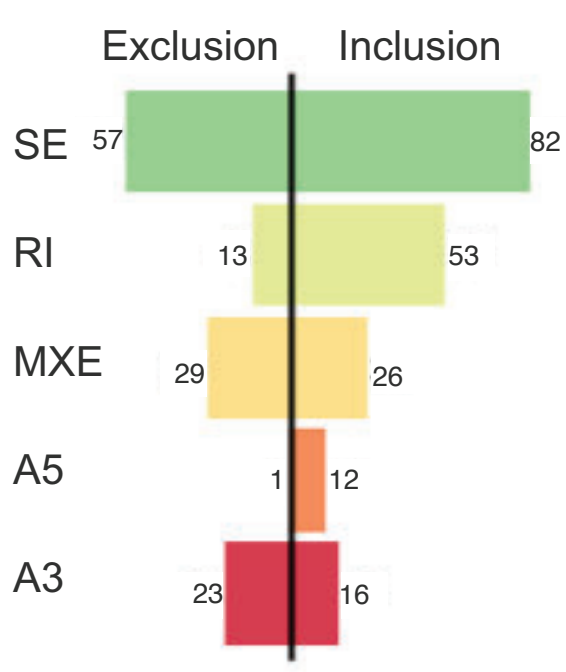

C

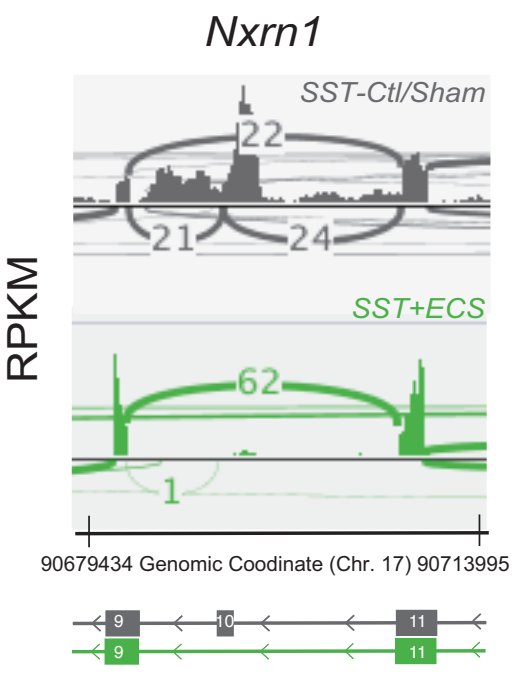

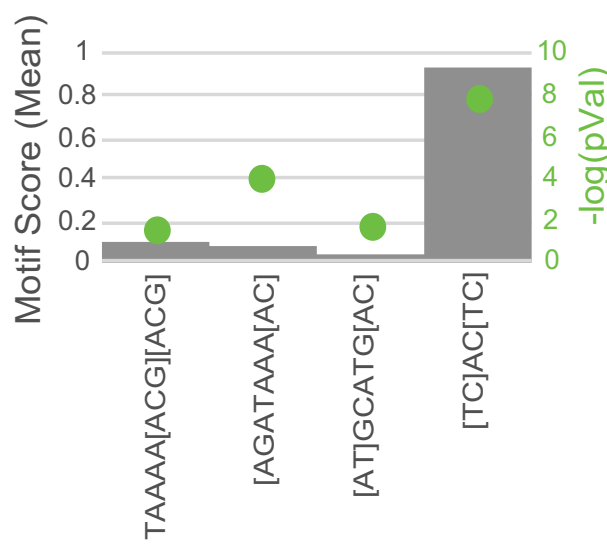

SAM68 SLM2 Rbfox1 Nova1/2

E

\section{$\mathbf{F}$}

synapse maturation (GO:0060074)
regulation of protein localization to membrane (GO:1905475) positive regulation of synaptic transmission (GO:0050806) ubiquitin-dependent protein catabolic process (GO:2000058) axon guidance (GO:0007411) neuron projection guidance (GO:0097485) regulation of axonogenesis (GO:0050770) axonogenesis (GO:0007409) regulation of developmental growth (GO:0048638) anterograde trans-synaptic signaling (GO:0098916)

chemical synaptic transmission (GO:0007268) morphogenesis involved in differentiation (GO:0010769) cognition (GO:0050890)

trans-synaptic signaling (GO:0099537) synaptic signaling (GO:0099536) modulation of chemical synaptic transmission (GO:0050804)regulation of trans-synaptic signaling (GO:0099177)

axon development (GO:0061564) regulation of neuron death (GO:1901214)neuron projection morphogenesis (GO:0048812) cell projection morphogenesis (GO:0120039) cell part morphogenesis (GO:0032990)

regulation of cell growth (GO:0001558) neuron projection development (GO:0031175)

protein phosphorylation (GO:0006468)

cell projection organization (GO:0030030)

cellular protein localization (GO:0034613) protein modification process (GO:0036211)

regulation of signaling (GO:0023051) sensory perception of smell (GO:0007608)-

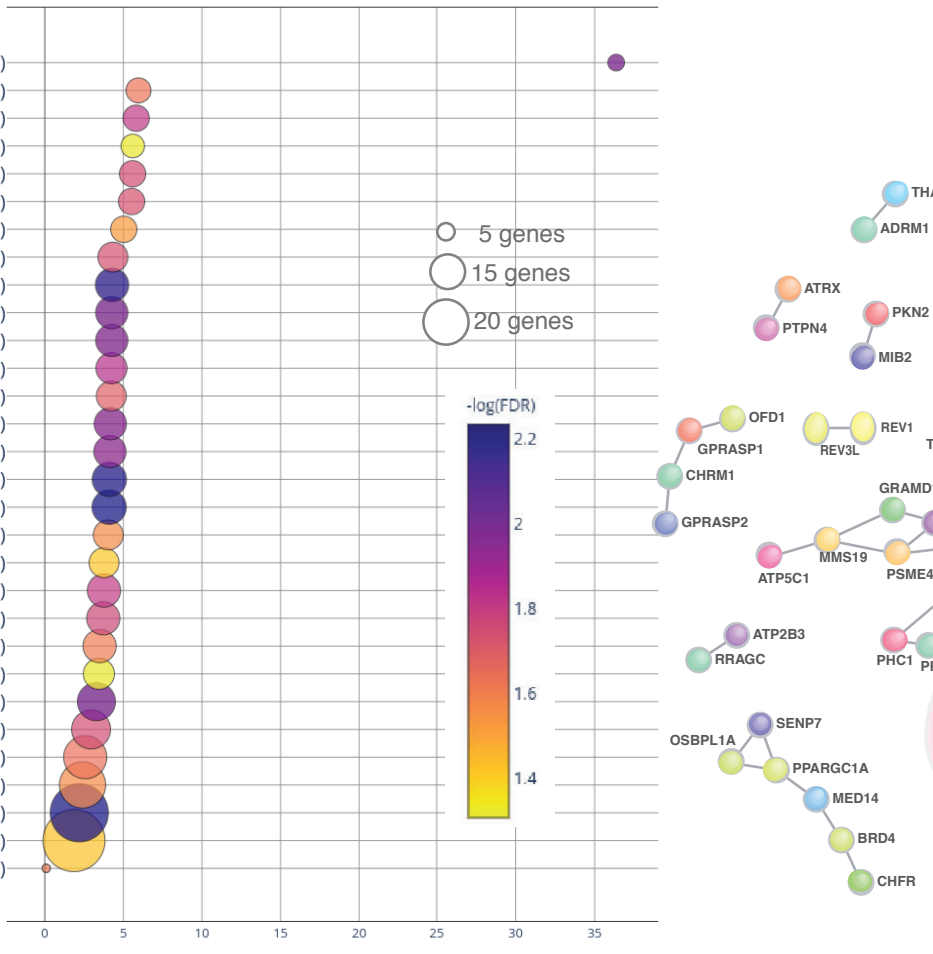

Percentage AS-genes within category

\section{SST cIN post-synaptic}

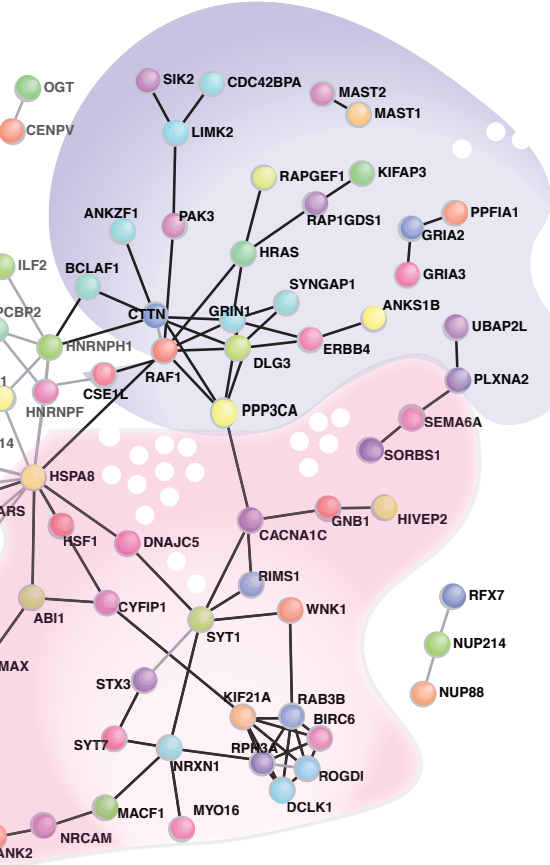

SST cIN pre-synaptic 
bioRxiv preprint doi: https://doi.org/10.1101/845230; this version posted November 16, 2019. The copyright holder for this preprint (which was

A

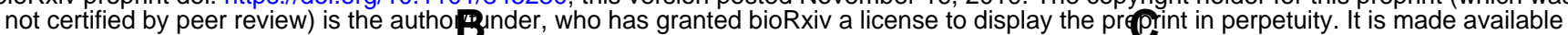
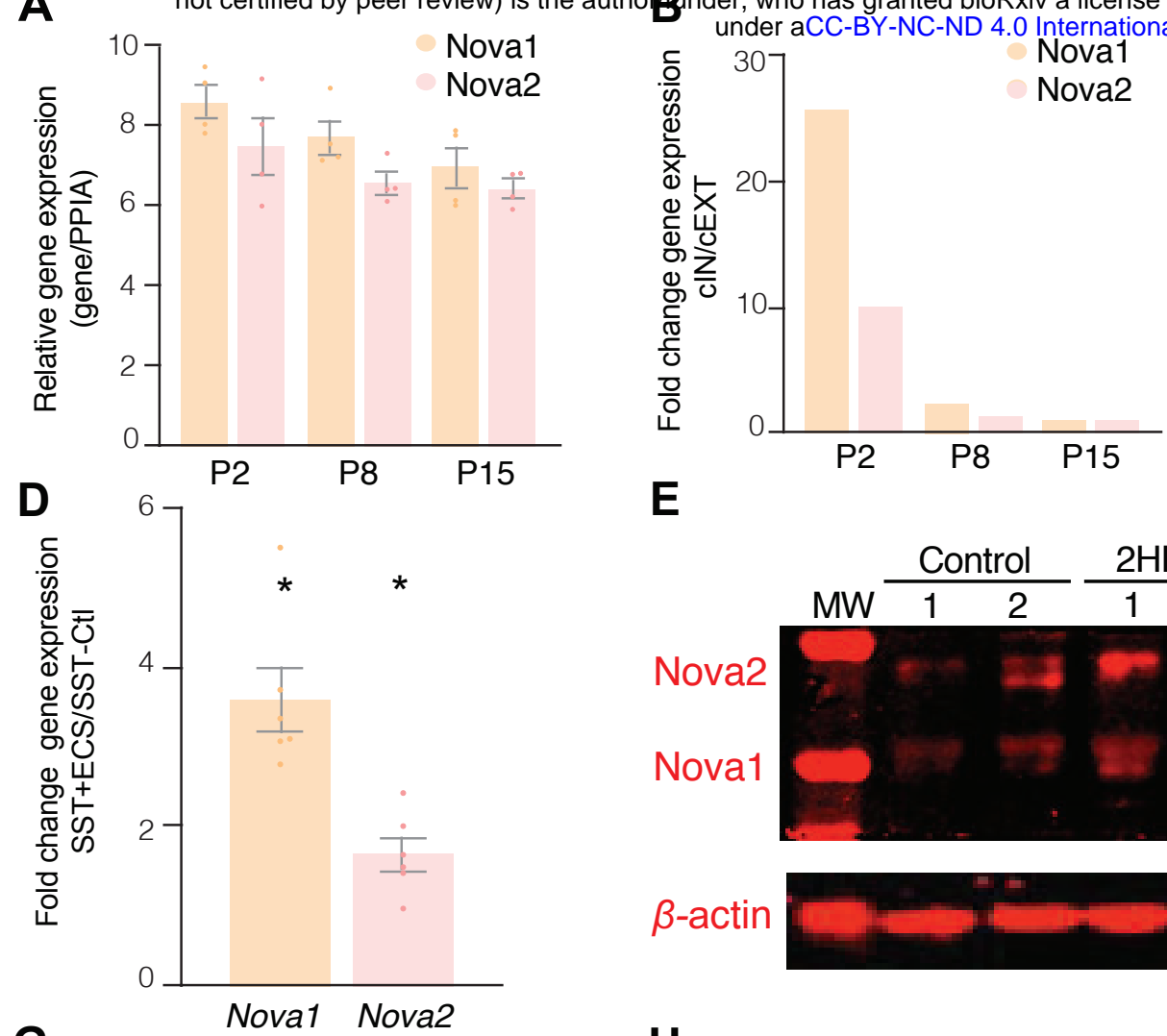

G
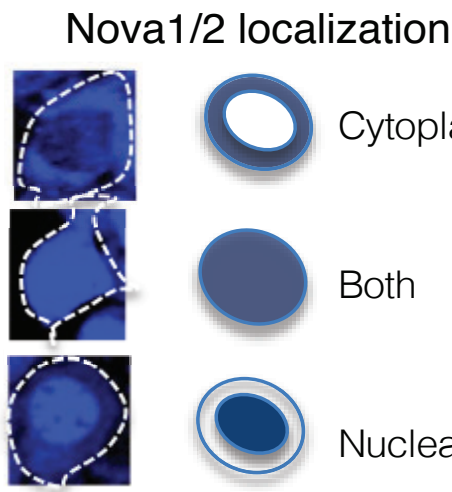

Cytoplasm

Both

Nuclear

$\mathbf{E}$

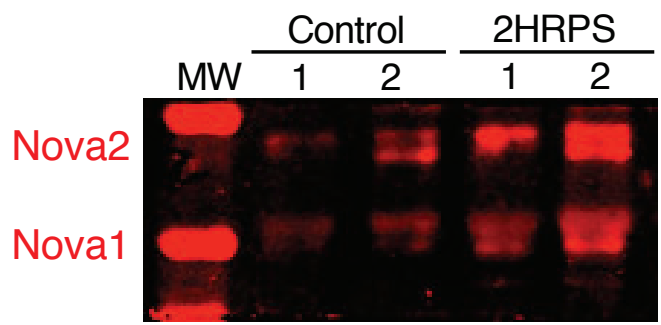

$\beta$-actin

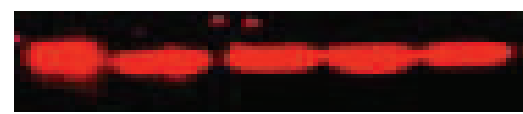

H
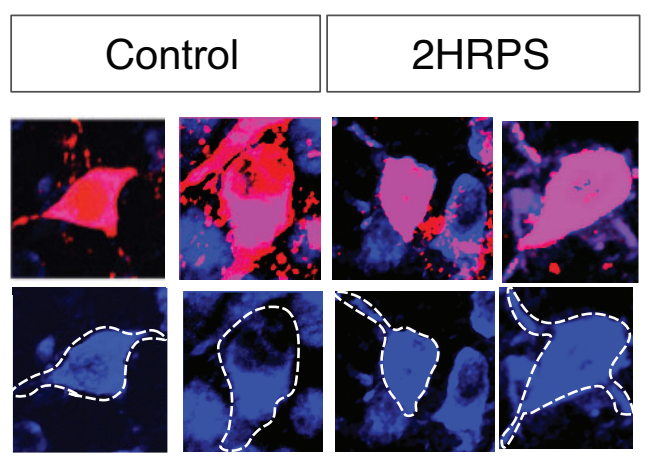

Acute Activity Induction P8

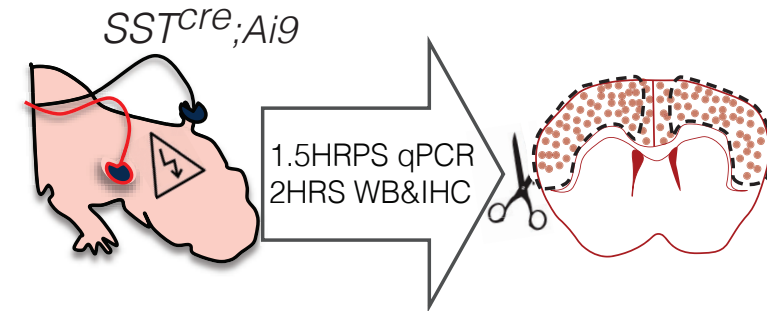

$\mathbf{F}$

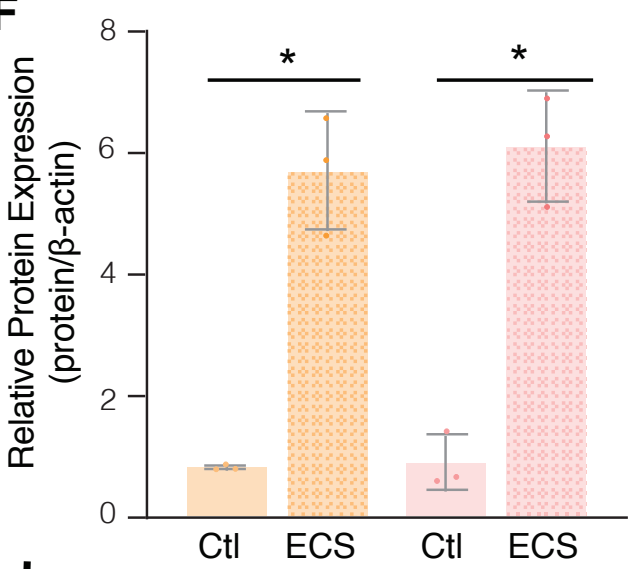

I

K
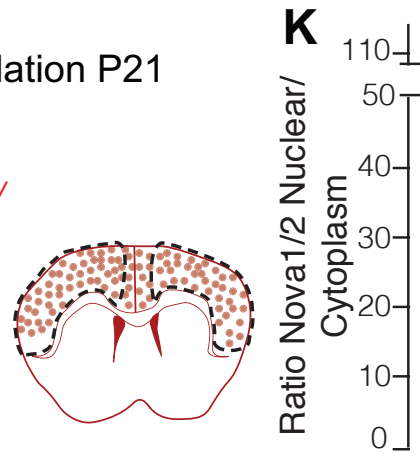
AAV-Flex-KIR2.1-mCherry AAV-Flex-NaChbac-mCherry AAV-Flex-mCherry

SSTCre

P0-P21

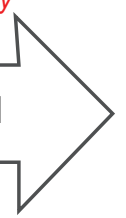

0

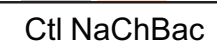

N

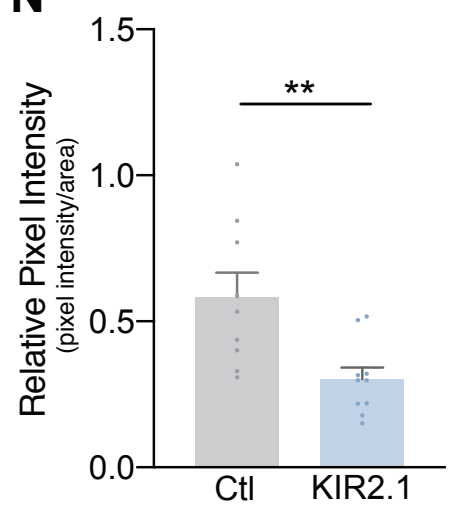

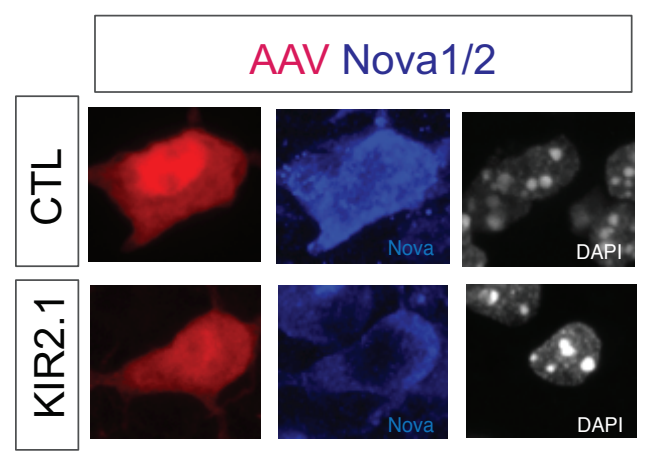


bioRxiv preprint doi: https://doi org/10.1101/845230; this version posted November 16,2019 . The copyright holder for this preprint (which was not certified by peer review) is the author/funder, who has granted bioRxiv a license to display the preprint in perpetuity. It is made available

A SSTCre; Nova1f/f;Nova2f/f;Ai9 or under aCC-BY-NC-ND 4.0 International license. SSTCre;Aig control
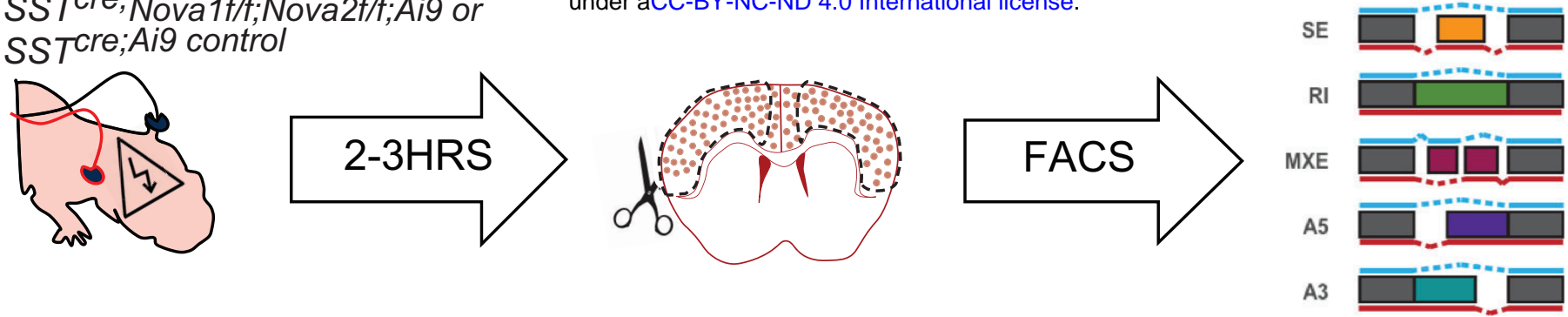

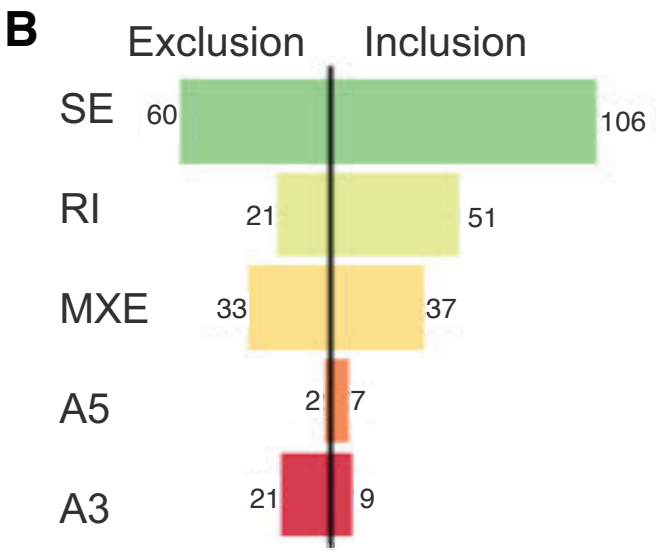

C

E

presynaptic dense core vesicle exocytosis (GO:0099525) synaptic vesicle priming (GO:0016082) dendritic spine morphogenesis (GO:0060997) dendritic spine organization (GO:0097061) protein localization to synapse (GO:0035418) receptor localization to synapse (GO:0097120) regulation of neuronal synaptic plasticity (GO:0048168) glutamate receptor signaling pathway (GO:0007215) neuron projection organization (GO:0106027) neurotransmitter secretion (GO:0007269) signal release from synapse (GO:0099643) exocytic process (GO:0140029) postsynapse organization (GO:0099173) regulation of synaptic transmission, glutamatergic (GO:0051966) dendrite morphogenesis (GO:0048813) synapse organization (GO:0050808) synaptic vesicle localization (GO:0097479) positive regulation of dendrite development (GO:1900006) positive regulation of synaptic transmission (GO:0050806) regulation of synaptic plasticity (GO:0048167) synaptic vesicle transport (GO:0048489) establishment of synaptic vesicle localization (GO:0097480) synaptic vesicle cycle (GO:0099504) regulation of dendrite development (GO:0050773) neurotransmitter transport (GO:0006836) signal release (GO:0023061)

chemical synaptic transmission (GO:0007268) anterograde trans-synaptic signaling (GO:0098916) trans-synaptic signaling (GO:0099537) synaptic signaling (GO:0099536)

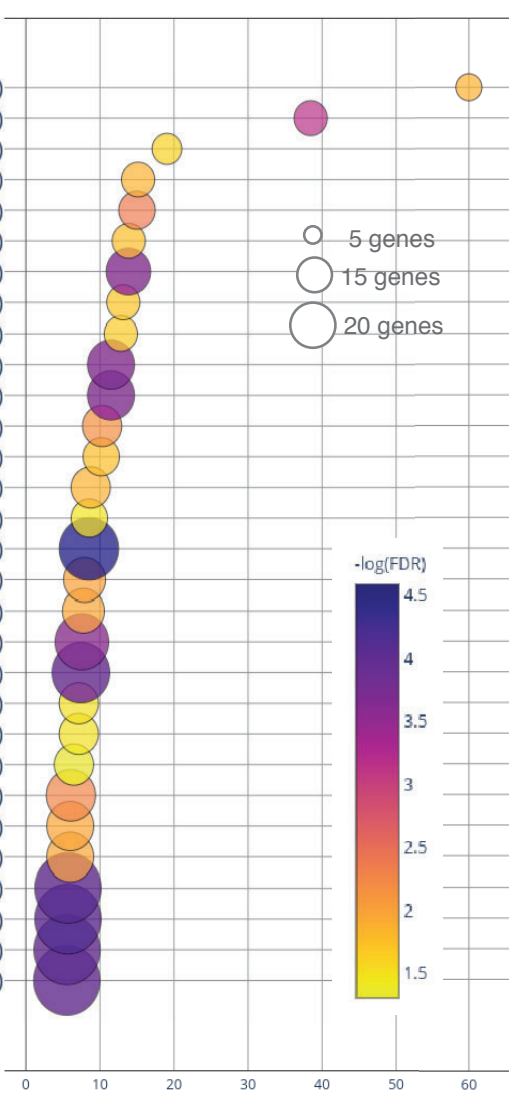

$\mathbf{F}$

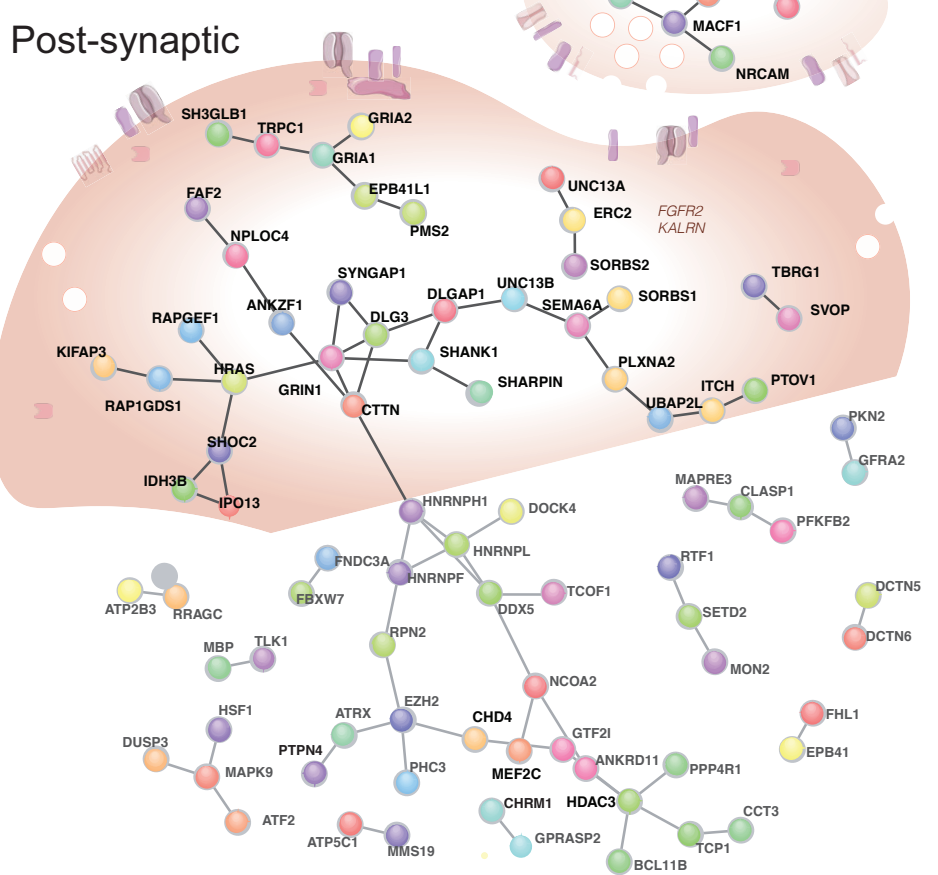

Pre-synaptic

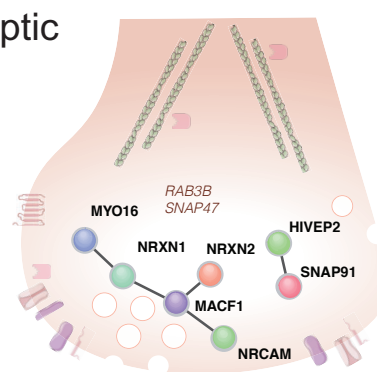

Syngap1

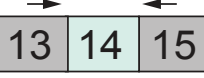

$13 \mid 15$
247

205

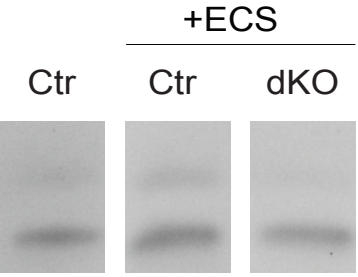

D

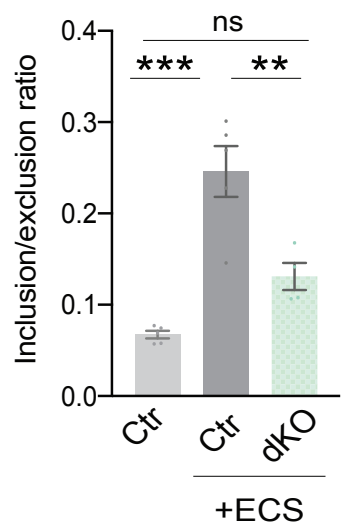

Percentage AS-genes within category 
Genetic Strategy:

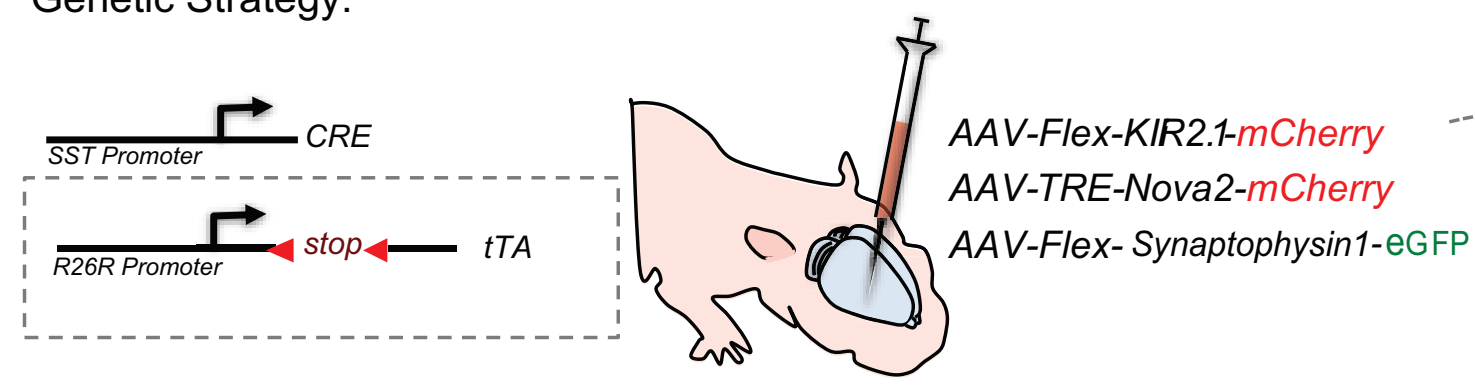

\section{SYPeGFP}

B
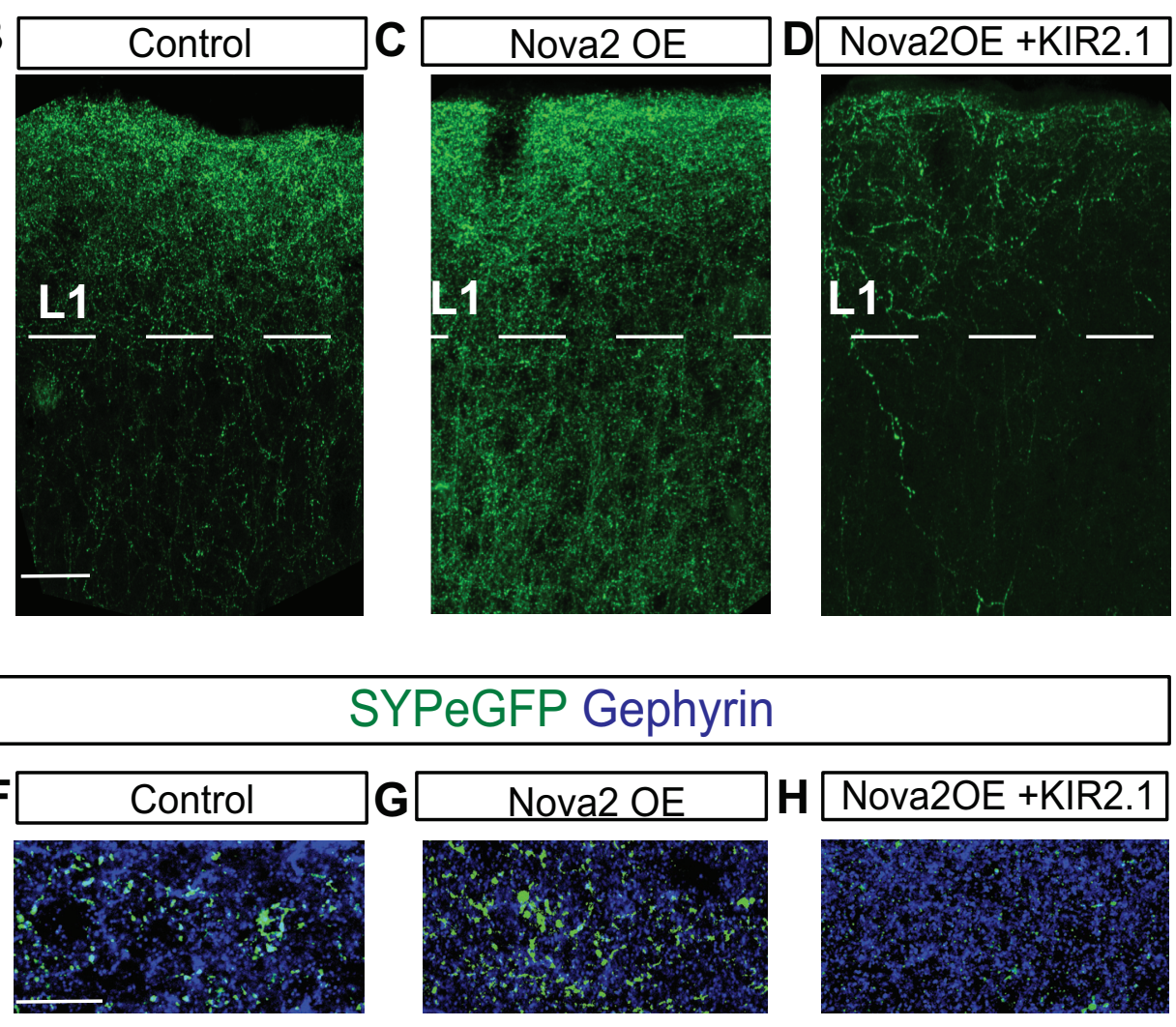

SYPeGFP Gephyrin

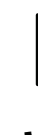

$$
\text { J }
$$

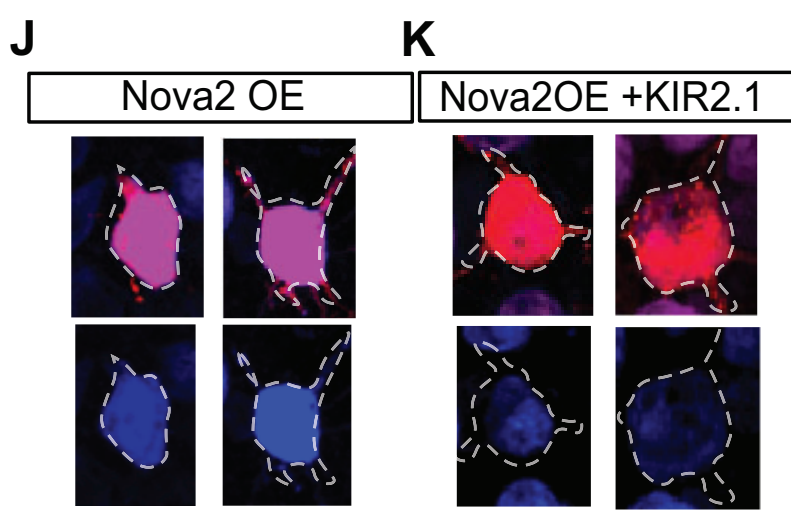

AAV Nova1/2
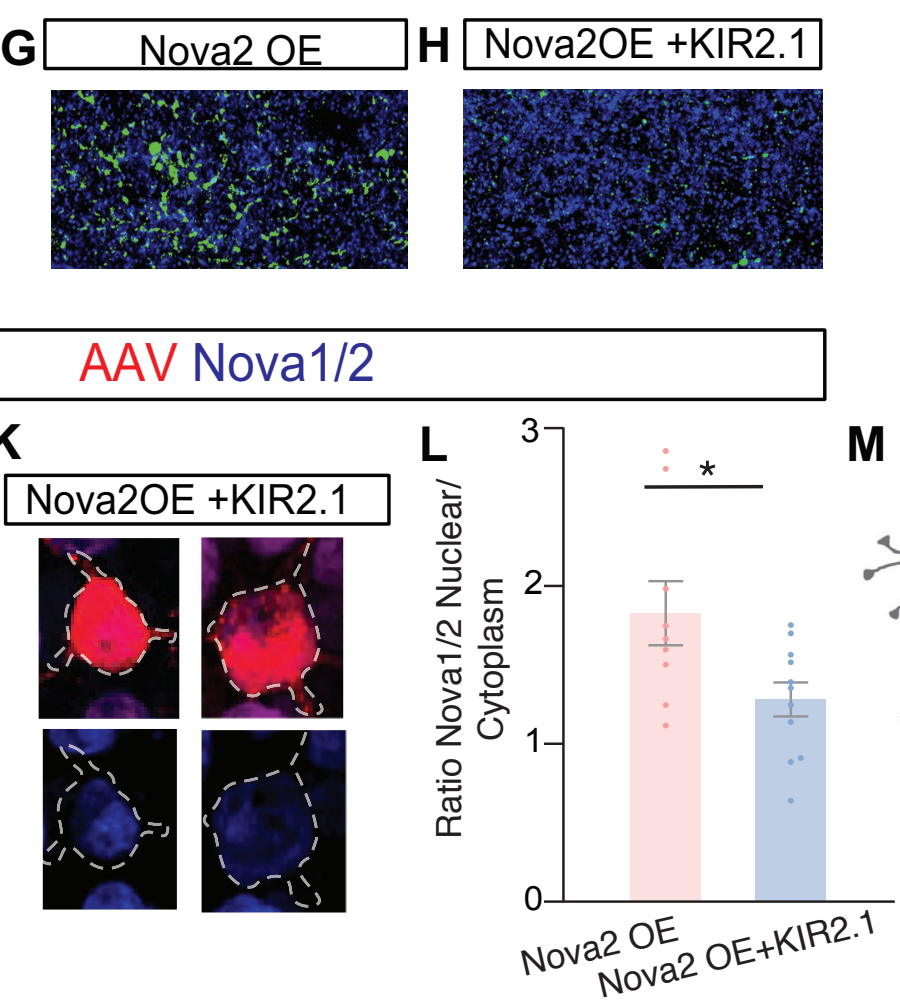

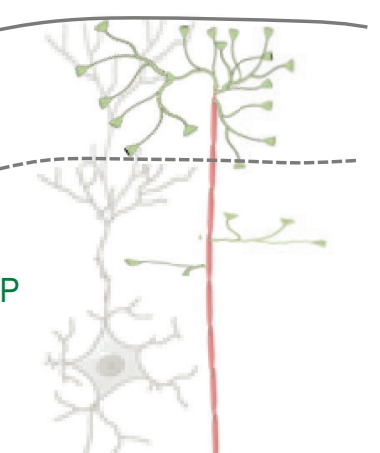

SST+CINs
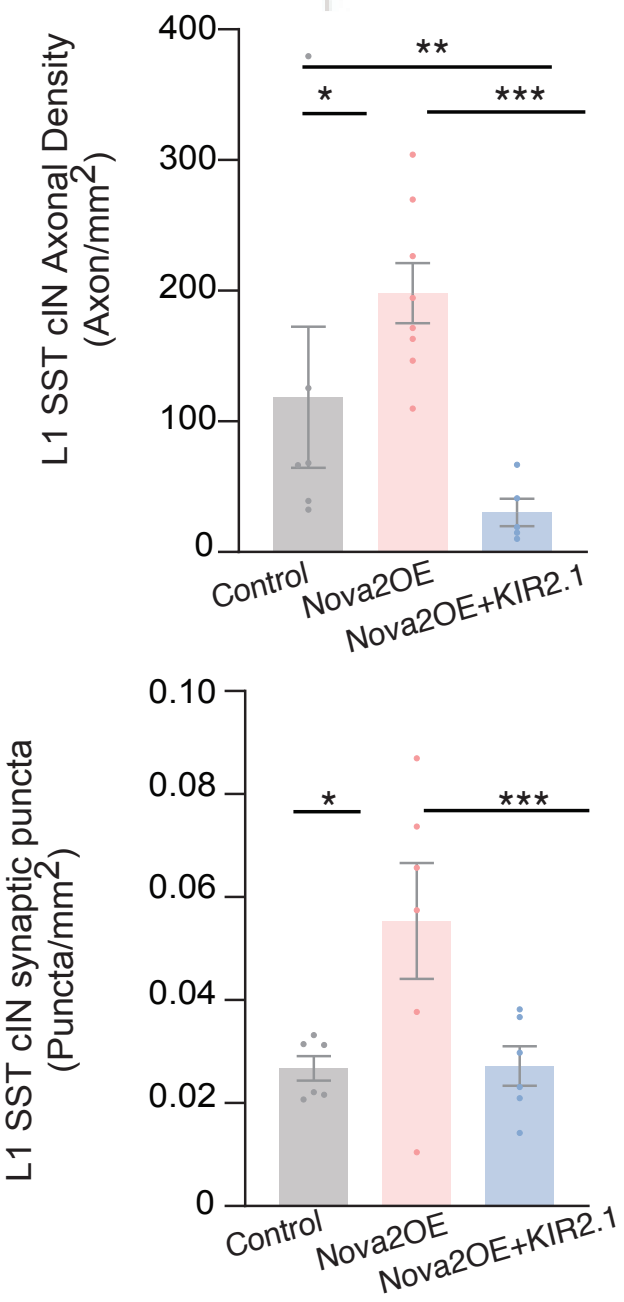

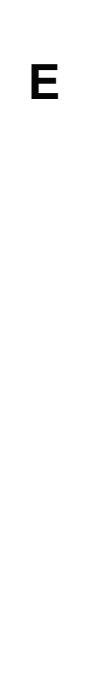

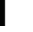

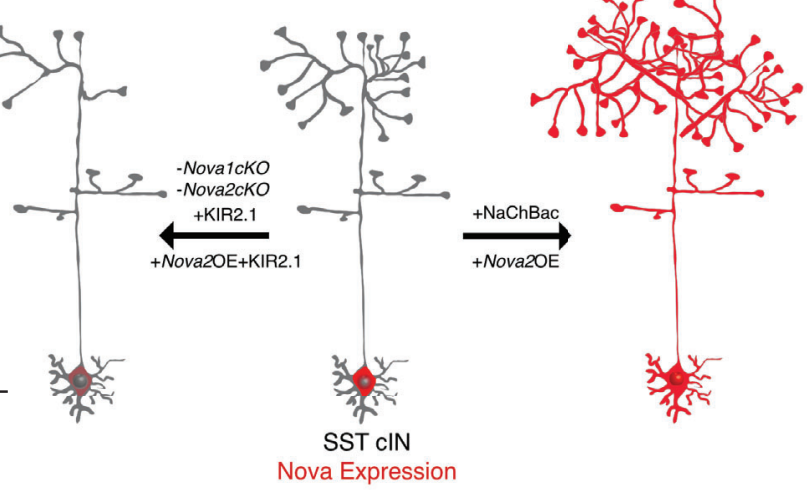

